Mon. Not. R. Astron. Soc. 000, 000-000 (0000) Printed 25 January $2021 \quad$ (MN LATEX style file v2.2)

\title{
Reionization and galaxy inference from the high-redshift Ly $\alpha$ forest
}

\author{
Yuxiang Qin ${ }^{1 \star}$, Andrei Mesinger ${ }^{1}$, Sarah E. I. Bosman ${ }^{2,3}$ and Matteo Viel ${ }^{4,5,6,7}$ \\ ${ }^{1}$ Scuola Normale Superiore, Piazza dei Cavalieri 7, I-56126 Pisa, Italy \\ ${ }^{2}$ Department of Physics and Astronomy, University College London, Gower Street, London WC1E 6BT, UK \\ ${ }^{3}$ Max-Planck-Institut für Astronomie, Königstuhl 17, D-69117 Heidelberg, Germany \\ ${ }^{4}$ SISSA - International School for Advanced Studies, via Bonomea 265, 34136 Trieste, Italy \\ ${ }^{5}$ IFPU, Via Beirut 2, 34014, Trieste, Italy \\ ${ }^{6}$ INFN, sezione di Trieste, via Valerio 2, Trieste, Italy \\ ${ }^{7}$ INAF - Osservatorio Astronomico di Trieste, Via G.B. Tiepolo 11, I-34131 Trieste, Italy
}

25 January 2021

\begin{abstract}
The transmission of Lyman- $\alpha(\operatorname{Ly} \alpha)$ in the spectra of distant quasars depends on the density, temperature, and ionization state of the intergalactic medium (IGM). Therefore, high-redshift $(z>5)$ Ly $\alpha$ forests could be invaluable in studying the late stages of the epoch of reionization (EoR), as well as properties of the sources that drive it. Indeed, high-quality quasar spectra have now firmly established the existence of large-scale opacity fluctuations at $z>5$, whose physical origins are still debated. Here we introduce a Bayesian framework capable of constraining the EoR and galaxy properties by forward-modelling the high- $z$ Ly $\alpha$ forest. Using priors from galaxy and CMB observations, we demonstrate that the final overlap stages of the EoR (when $>95 \%$ of the volume was ionized) should occur at $z<5.6$, in order to reproduce the large-scale opacity fluctuations seen in forest spectra. However, it is the combination of patchy reionization and the inhomogeneous UV background that produces the longest Gunn-Peterson troughs. Ly $\alpha$ forest observations tighten existing constraints on the characteristic ionizing escape fraction of galaxies, with the combined observations suggesting $f_{\mathrm{esc}} \approx 7_{-3}^{+4} \%$, and disfavoring a strong evolution with the galaxy's halo (or stellar) mass.
\end{abstract}

Key words: cosmology: theory - dark ages, reionization, first stars - diffuse radiation - early Universe - galaxies: high-redshift - intergalactic medium

\section{INTRODUCTION}

The presence of residual neutral hydrogen in the intergalactic medium (IGM), leads to a series of absorption lines in the spectra of distant galaxies and quasars, corresponding to photons redshifting into Lyman- $\alpha(\mathrm{Ly} \alpha)$ resonance (Savaglio et al. 2002, Lee et al. 2014; Faucher-Giguère et al. |2008b, Busca et al. 2013. Lee et al. 2013; Slosar et al. 2013 | Iršič et al. 2017). This so-called Ly $\alpha$ forest provides invaluable insights into the structure and properties of the high-redshift IGM (e.g. Bolton et al. 2010, Lidz et al. 2010, Garzilli et al. 2012, Lee et al.| 2015; Puchwein et al. 2015, Bolton et al. 2017 Gaikwad et al. 2020), the cosmic radiation fields that regulate them (e.g. Bolton \& Haehnelt 2007b; FaucherGiguère et al. 2008a, Becker \& Bolton 2013, D'Aloisio et al. 2018), as well as physical cosmology (e.g. Miralda-Escudé et al. 1996, Croft et al. 2002, Viel et al. 2005, 2013, Delubac et al. 2015, Bautista et al. 2017; Yèche et al. 2017).

Of particular note is the potential of the Ly $\alpha$ forest in studying the observationally-starved epoch of reionization (EoR). Indeed historically, the Ly $\alpha$ forest provided the first constraint on the ionization state of our Universe (Gunn \& Peterson 1965).

Recent years have witnessed a large increase in the number of high-quality, high-redshift $(z \gtrsim 5-6)$ quasar spectra (e.g. Fan et al. 2006 Becker et al. 2007, Willott et al. 2010 Mortlock et al. 2011; Venemans et al. 2013, Wu et al. 2015 Becker et al. 2015, Jiang et al. 2016 Bañados et al. 2018 Yang et al. 2020). These have been used to search for increasingly subtle EoR signatures. Redward of the Ly $\alpha$ emission line, an incomplete EoR can be studied through absorption from the Ly $\alpha$ damping-wing profile (Bolton et al. 2011. Schroeder et al. 2013, Davies et al. 2018b Greig et al. 2017, 2019. Wang et al. 2020), while on the blueward 
Qin et al.

side, the additional ionizing contribution from the quasar itself facilitates Ly $\alpha$ transmission in the so-called near zone (e.g. Mesinger et al. 2004, Bolton \& Haehnelt 2007a| Lidz et al. 2007; Maselli et al. 2007, Eilers et al. 2017, 2020, Davies et al. 2020). Blueward of the quasar near zone, the Ly $\alpha$ forest becomes more opaque and trace amounts of HI are sufficient to saturate transmission.

However, some transmission in the forest is seen even at the highest redshifts. In fact, the sightline-to-sightline scatter in the transmission has been suggested as a potential probe of the EoR (e.g. Becker et al. 2007. Gallerani et al. 2008, Bosman et al. 2018, Eilers et al. 2018). The significant scatter recently observed on large scales (tens hundred $\mathrm{cMpc}$ ) is especially promising. For example, the $110 h^{-1} \mathrm{cMpc}$ Gunn-Peterson trough observed in ULAS J0148+0600 cannot be explained by fluctuations in the gas density alone (e.g. Becker et al. 2015).

There have been several theoretical explanations for the sizeable sightline-to-sightline fluctuations, focusing on (i) gas temperature (e.g. D'Aloisio et al. 2015, Keating et al. 2018); (ii) rare sources (Chardin et al. 2015, 2017||D'Aloisio et al. 2017 Meiksin 2020); (iii) the mean free path of ionizing UV photons (Davies \& Furlanetto 2016, D'Aloisio et al. 2018); and (iv) late reionization (Kulkarni et al. 2019; Keating et al. 2020b; Nasir \& D'Aloisio 2020).

Unfortunately, there are significant degeneracies between these models (see e.g. Nasir \& D'Aloisio 2020), and most previous work has been fairly qualitative - showing a handful of models which agree with the observations to various degrees. Robust, quantitative constraints require an exhaustive Bayesian inference framework. For instance, Choudhury et al. (2021) recently used semi-numerical simulations to constrain the EoR history using forward-modelled cumulative distribution functions (CDFs) of the effective optical depth $\tau_{\text {eff }}$. Their seminal work, appearing as this paper was nearing completion, used effective parameters to approximate inhomogeneous recombinations, in addition to assuming a constant mass-to-light ratio for galaxies.

Here, we showcase a fully Bayesian framework for interpreting the Ly $\alpha$ forest at high redshift using $21 \mathrm{cmFAsT}^{1}$ (Mesinger \& Furlanetto 2007, Mesinger et al. 2011, Murray et al. 2020;) and its Markov chain Monte Carlo (MCMC) driver, 21CMMC ${ }^{2}$ (Greig \& Mesinger 2015, 2017). Building on the model introduced by Park et al. (2019), we directly sample galaxy properties and forward-model the 3D lightcone of Ly $\alpha$ transmission. Our galaxy-driven model allows us to fold-in observations of high-redshift UV luminosity functions (LFs; Bouwens et al. 2015, 2016, Oesch et al. 2016), in addition to EoR constrains from the Thomson scattering optical depth $\left(\tau_{e} ;\right.$ Planck Collaboration et al. 2020) and quasar dark fraction measurements (McGreer et al. 2015). All codes developed here are publicly available.

This paper is organized as follows. We present our model including galaxy properties, reionization, IGM temperature and Ly $\alpha$ forests in Section 2. We then summarize the observed forest sample and free parameters used for inference in Sections 3 and 4 . We present our inference results in Section 5 before concluding in Section 6. We assume

\footnotetext{
1 https://github.com/21cmfast/21cmFAST
}

2 https://github.com/21cmfast/21CMMC a $\Lambda$ CDM cosmology with parameters $\left(\Omega_{\mathrm{m}}, \Omega_{\mathrm{b}}, \Omega_{\Lambda}, h, \sigma_{8}, n_{s}\right.$ $=0.31,0.049,0.69,0.68,0.81,0.97)$ chosen from the $T T, T E, E E+$ lowE + lensing $+B A O$ reconstruction in Planck Collaboration et al. (2020).

\section{MODELLING GALAXIES, THE IGM AND Ly $\alpha$ TRANSMISSION}

We first summarize our parametrization of high-redshift galaxies, whose cosmic radiation fields govern the evolution of the high-redshift IGM. We then discuss the corresponding IGM properties, including inhomogenous reionization, local recombination and photoionization rates, before presenting the addition of two new outputs of 21cmFAsT developed in this work: post-reionization gas temperature and the Ly $\alpha$ optical depth.

\subsection{High-redshift galaxies}

We adopt the galaxy model of Park et al. (2019), which relates bulk galaxy properties to the halo mass function through power-law scalings. Such a parametrization can recover high-redshift galaxy UV LFs (see also, e.g. Moster et al. 2013, Sun \& Furlanetto 2016, Mutch et al. 2016, Tacchella et al. |2018, Behroozi et al. |2019, Yung et al.| 2019). Therefore, we can use galaxy observations, in addition to the Ly $\alpha$ forest, to constrain our model parameters. This improves on some previous forest studies (e.g. Mesinger \& Furlanetto 2009 Choudhury et al. 2021) that assumed a constant mass to light ratio, and allows us to use physicallymeaningful priors when performing inference (e.g. ionizing escape and stellar mass fractions must be between 0 and 1).

Specifically, the number density of galaxies is described by the halo mass function, $\mathrm{d} n / \mathrm{d} M_{\mathrm{vir}}$, with an additional factor of $\exp \left(-M_{\text {turn }} / M_{\text {vir }}\right)$ accounting for inefficient star formation in low-mass halos due to ineffective cooling, inhomogeneous feedback from reionization photo-heating and/or supernova feedback (Efstathiou 1992, Shapiro et al. 1994, Thoul \& Weinberg 1996| Hui \& Gnedin 1997. Sobacchi \& Mesinger 2014; Hopkins et al. 2014 , Wyithe \& Loeb 2013; Sun \& Furlanetto 2016; Mutch et al. 2016 Hopkins et al. 2018). The average stellar mass of a galaxy hosted by a halo of mass $M_{\text {vir }}$ can be written as $M_{*}=M_{\mathrm{vir}} \Omega_{\mathrm{b}} / \Omega_{\mathrm{m}} \times \min \left[1, f_{*, 10}\left(M_{\mathrm{vir}} / 10^{10} \mathrm{M}_{\odot}\right)^{\alpha_{*}}\right]$. Similarly, the UV ionizing escape fraction is taken to be $f_{\text {esc }}=\min \left[1, f_{\text {esc }, 10}\left(M_{\text {vir }} / 10^{10} \mathrm{M}_{\odot}\right)^{\alpha_{\text {esc }}}\right]$. Assuming the characteristic star formation timescale is proportional to the halo dynamical timescale, we take $M_{*} / \dot{M}_{*}=t_{*} H^{-1}$ where $H(z)$ represents the Hubble parameter at $z$. We can then estimate the non-ionizing UV luminosity through $L_{1500}=\dot{M}_{*} \times 8.7 \times 10^{27} \mathrm{erg} \mathrm{s}^{-1} \mathrm{~Hz}^{-1} \mathrm{M}_{\odot}^{-1}$ yr Madau \& Dickinson 2014) and compare against high-redshift observations (e.g. Finkelstein et al. 2015. Bouwens et al. 2015, 2016, Livermore et al. |2017 | Atek et al.||2018, Ishigaki et al.|2018, Oesch et al. 2018, Bhatawdekar et al. 2019).

We thus have 6 free parameters to characterize the UV ionizing properties of high-redshift galaxies: $M_{\mathrm{turn}}, f_{*, 10}, \alpha_{*}$, $f_{\text {esc }, 10}, \alpha_{\text {esc }}$ and $t_{*}$. In the next subsection, we summarize how we calculate the IGM properties corresponding to a given galaxy model. 


\subsection{The IGM}

We begin by generating a Gaussian realization of $\Lambda \mathrm{CDM}$ initial conditions in a periodic box with a side length of $500 \mathrm{cMpc}$ and a cell resolution of $\sim 0.39 \mathrm{cMpc}$ (i.e. $500 \mathrm{cMpc} / 1280)$. Using second-order Lagrangian perturbation theory (Scoccimarro 1998) with high-res velocity and density fields ${ }^{3}$ we evolve these fields towards lower redshifts and re-grid them onto a lower resolution box $(\sim 1.95 \mathrm{cMpc}$; i.e. $500 \mathrm{cMpc} / 256)$ to calculate ionization fields.

\subsubsection{Inhomogeneous reionization}

Cosmological reionization by UV photons is effectively bimodal, with ionized regions surrounding galaxies expanding and eventually overlapping to complete reionization. EoR models need to track this inhomogenous process, as well as estimate the residual $\mathrm{HI}$ inside the ionized component of the IGM. We summarize our procedures for these in turn.

To identify ionized cells, we use an excursion-set ap$\operatorname{proach}^{4}($ Furlanetto et al. 2004). Centered on a cell at $(\mathbf{r}, z)$, we consider spherical volumes with decreasing radii, $R$, and corresponding overdensities, $\delta_{\left.\mathrm{R}\right|_{\mathbf{r}, \mathrm{z}}} \equiv\left\langle\rho_{\mathrm{b}} / \bar{\rho}_{\mathrm{b}}-1\right\rangle_{R}$ where $\rho_{\mathrm{b}}$ and $\bar{\rho}_{\mathrm{b}}$ are the baryon density and its cosmic mean. Using the corresponding conditional halo mass function Barkana \& Loeb 2005, Mesinger et al. 2011), we compute the cumulative number of ionizing photons per baryon in this spherical IGM patch by

$\bar{n}_{\text {ion }}=\int \mathrm{d} M_{\text {vir }} \frac{\mathrm{d} n}{\mathrm{~d} M_{\text {vir }}} \exp \left(-\frac{M_{\text {turn }}}{M_{\text {vir }}}\right) M_{*} \rho_{\mathrm{b}}^{-1} n_{\gamma} f_{\text {esc }}$,

where $n_{\gamma}=5000$ is the number of ionizing photons intrinsically emitted per stellar baryon (Barkana \& Loeb 2005).

We follow Sobacchi \& Mesinger (2014) to estimate the average number of recombinations per baryon, $\bar{n}_{\text {rec }}$. Using the probability distribution function (PDF; $\left.\mathrm{d} n / \mathrm{d} \rho_{\mathrm{sub}}\right)$ of sub-grid (unresolved by our simulation cell $\lesssim 1.95 \mathrm{cMpc}$ ) densities $\left(\rho_{\text {sub }}\right)$ from Miralda-Escudé et al. (2000), adjusted for the mean density in the cell, we calculate the recombination rate by

$\dot{n}_{\mathrm{rec}}=\int \mathrm{d} \rho_{\mathrm{sub}} \frac{\mathrm{d} n}{\mathrm{~d} \rho_{\mathrm{sub}}} \alpha_{\mathrm{B}} f_{\mathrm{H}} \rho_{\mathrm{b}}^{-1} \rho_{\mathrm{sub}}^{2}\left(1-x_{\mathrm{HI}}\right)^{2}$,

3 Rather than evolving the density field using lower resolution velocity fields (which was originally implemented in $21 \mathrm{cmFAST}$ to conserve RAM, given that velocity fields have much longer correlation lengths), here we use high-resolution for all of the initial conditions (setting PERTURB_ON_HIGH_RES=TRUE). This was included to guarantee the density fields are as accurate as possible for simulating the Ly $\alpha$ forest (Watkinson et al., in prep).

4 Excursion-set algorithms generally do not conserve photons when Hiı bubbles overlap (e.g. Zahn et al. 2007 Paranjape \& Choudhury 2014). In practice, this translates to a bias in the effective ionizing escape fraction (e.g. Hutter 2018). Using the updated, photon-conserving algorithm of 21cmFAST v3, we quantify that this is a very minor effect for our model parametrization, resulting in a bias of $\sim-0.2$ for $\alpha_{\text {esc }}$ (Park et al., in prep). In other words, the recovered posterior without ionizing photon conservation differs from the true posterior including ionizing photon conservation primarily through a translation in one of the parameters: $\alpha_{\mathrm{esc}}^{\text {true }} \rightarrow \alpha_{\mathrm{esc}}^{\text {recovered }}+0.2$. However, as photon-conservation slows down our calculation by a factor of $\sim 2$, we leave this option off in this proof-of-concept study, highlighting the resulting bias in the marginalized posterior of $\alpha_{\text {esc }}$ (see more in Section 5 ). where $\alpha_{\mathrm{B}}, f_{\mathrm{H}}$ and $x_{\mathrm{HI}}$ are the case-B recombination coefficient 5 number fraction of hydrogen in the Universe, and the (residual) neutral hydrogen fraction of the sub-grid gas element, respectively. The calculation of $x_{\mathrm{HI}}$ is presented in Section 2.2.2.

The cell is then considered to be ionized if the cumulative number of ionizing photons is larger than the number of baryons plus recombinations. Specifically, the cell is considered as ionized if at any radius, $R$,

$\bar{n}_{\text {ion }}-1 \geqslant \bar{n}_{\text {rec }}=\left\langle\int_{z_{\text {ion }}}^{z} \mathrm{~d} z^{\prime} \frac{\mathrm{d} t}{\mathrm{~d} z^{\prime}} \dot{n}_{\mathrm{rec}}\right\rangle_{R}$,

where $z_{\text {ion }}$ is the reionization redshift of a cell and \langle\rangle$_{R}$ denotes averaging over all cells within the spherical HiI region. We also approximate the local mean free path $\left(R_{\mathrm{MFP}}\right)$ using the largest radius at which this equation is valid. This is strictly true in the early stages of reionization that are not affected by IGM recombinations, but should also be a good approximation for the overlap stages as they likely evolve in a "photon-starved" manner (Bolton \& Haehnelt 2007b Sobacchi \& Mesinger 2014). In future work, we will generalize this derivation, which will allow us to extend our models to lower redshifts.

\subsubsection{Residual neutral hydrogen inside the ionized IGM}

Since a trace amount of neutral hydrogen can obscure all flux at the Ly $\alpha$ transition, it is important to determine the residual neutral hydrogen fraction within the cosmic His regions. Assuming photoionization equilibrium in the reionized IGM, we evaluate $x_{\mathrm{HI}}$ by solving

$x_{\mathrm{HI}} f_{\text {ion }, \mathrm{ss}} \Gamma_{\text {ion }}=\chi_{\mathrm{HeII}} n_{\mathrm{H}}\left(1-x_{\mathrm{HI}}\right)^{2} \alpha_{\mathrm{B}}$

where $\Gamma_{\text {ion }}$ is the local photoionization background $\sqrt{6} f_{\text {ion,ss }}$ is a self-shielding factor attenuating $\Gamma_{\text {ion }}, \chi_{\mathrm{HeII}}=1.08$ accounts for singly ionized helium, and $n_{\mathrm{H}}$ is the hydrogen number density in the cell.

The photoionization rates before self-shielding are estimated assuming a stellar-driven UV background (UVB) with a spectral index 7 of $\alpha_{\text {UVB }} \sim 5$ (Thoul \& Weinberg 1996)

$\Gamma_{\text {ion }}=(1+z)^{2} R_{\mathrm{MFP}} \sigma_{\mathrm{H}} \frac{\alpha_{\mathrm{UVB}}}{\alpha_{\mathrm{UVB}}+\beta_{\mathrm{H}}} \frac{\bar{\rho}_{\mathrm{b}}}{m_{\mathrm{p}}} \overline{\dot{n}}_{\mathrm{ion}}$,

where $\beta_{\mathrm{H}} \sim 2.75, m_{\mathrm{p}}$ and $\overline{\dot{n}}_{\text {ion }}$ are the spectral index of the

5 The recombination coefficient depends on the gas temperature $\left(T_{\mathrm{g}}\right), \alpha_{\mathrm{B}}=2.59 \times 10^{-10}\left(T_{\mathrm{g}} / \mathrm{K}\right)^{-0.75} \mathrm{~cm}^{3} \mathrm{~s}^{-1}$. For computational efficiency, we assume an average temperature of $T_{\mathrm{g}}=10^{4} \mathrm{~K}$ in equation 22 when computing the cumulative number of recombinations, used to identify if a region is ionized or not. We do account for local temperature fluctuations when computing the residual HI fraction inside the ionized IGM, as detailed in Section 2.2.2

6 Our model does not include small-scale fluctuations in $\Gamma_{\text {ion }}$, due to proximate galaxies. In most of the IGM, such Poisson fluctuations are negligible, and $\Gamma_{\text {ion }}$ is determined by the combined radiation from many galaxies (e.g. Mesinger \& Furlanetto 2009 Sadoun et al. 2017).

7 The effective spectral index for a stellar-driven UVB could be somewhat harder than we assume (e.g. Becker \& Bolton 2013 D'Aloisio et al. 2019); however, $\alpha_{\mathrm{UVB}}$ is degenerate with the ionizing escape fraction in equation 5 and we treat the later as a free parameter in our analysis. 
Qin et al.

Hi photoionization cross-section, the proton mass, and the mean production rate of ionizing photons evaluated using equation 11 with $M_{*}$ being replaced by $\dot{M}_{*}$. According to radiative transfer simulation results from Rahmati et al. (2013), the self-shielding factor depends on the local density $\left(\rho_{\mathrm{b}}\right)$, gas temperature $\left(T_{\mathrm{g}}\right)$ as well as the unattenuated photoionization rate $\left(\Gamma_{\text {ion }}\right)$ and follows

$f_{\text {ion }, \mathrm{ss}}=0.98\left[1+\left(\frac{\rho_{\mathrm{b}}}{\rho_{\mathrm{ss}}}\right)^{1.64}\right]^{-2.28}+0.02\left(1+\frac{\rho_{\mathrm{b}}}{\rho_{\mathrm{ss}}}\right)^{-0.84}$

where

$\rho_{\mathrm{ss}}=27 \bar{\rho}_{\mathrm{b}}\left(\frac{T_{\mathrm{g}}}{10^{4} \mathrm{~K}}\right)^{0.17}\left(\frac{\Gamma_{\mathrm{ion}}}{10^{-12} \mathrm{~s}^{-1}}\right)^{\frac{2}{3}}\left(\frac{1+z}{10}\right)^{-3}$

is the characteristic density for the onset of self-shielding (Schaye 2001).

\subsubsection{Gas temperature of the ionized IGM}

Inside the HiI regions, we calculate the inhomogeneous gas temperature in each simulation cell following McQuinn \& Upton Sanderbeck (2016):

$T_{\mathrm{g}}^{\gamma}=T_{\text {ion,I }}^{\gamma}\left[\left(\frac{\mathscr{Z}}{\mathscr{Z}_{\text {ion }}}\right)^{3} \frac{\rho_{\mathrm{b}}}{\rho_{\mathrm{b}, \text { ion }}}\right]^{\frac{2 \gamma}{3}} \frac{\exp \left(\mathscr{Z}^{2.5}\right)}{\exp \left(\mathscr{Z}_{\text {ion }}^{2.5}\right)}+T_{\text {lim }}^{\gamma} \frac{\rho_{\mathrm{b}}}{\bar{\rho}_{\mathrm{b}}}$

Here, $\mathscr{Z}$ denotes $(1+z) / 7.1, \gamma=1.7$ is the equation of state index, $T_{\text {ion,I }}$ represents the post I-front temperature, here taken to be a constant $\left(T_{\text {ion,I }}=2 \times 10^{4} \mathrm{~K}\right)^{8}$ and $T_{\text {lim }}=$ $1.775 \mathscr{Z} \times 10^{4} \mathrm{~K}$ refers to the final relaxation temperature. The subscript "ion" indicates the quantity is at the redshift of ionization, $z_{\text {ion. }}$. The two terms on the RHS of equation (8) correspond to the initial and final temperatures of photoionized IGM.

The memory of the initial, post-reionization temperature fades within $\Delta z \sim 1-2$ (see the exponential term in equation 8), after which the IGM approaches an equilibrium temperature resulting from the balance between photoheating and various cooling processes (i.e. Hubble expansion, recombination, Compton scattering and free-free radiation). Because reionization is "inside-out" on large scales (e.g. see the review of Trac \& Gnedin 2011), the underdense regions of the IGM reionize late. Thus, large-scale low-density regions at $z \sim 5-6$ should be hotter (due to the first term on the RHS of equation 8), resulting in a lower recombination rate coefficient in equation (4) and a corresponding increase in the Ly $\alpha$ transmission in the forest. On the other hand, the equilibrium temperature results in a temperature-density relation in which underdense regions are colder (see the second term on the RHS of quation 8, see also Hui \& Gnedin 1997), and have a correspondingly lower Ly $\alpha$ transmission. However, when averaged over large scales, only the former effect remains. Therefore, one would expect voids to correspond to large-scale peaks in the Ly $\alpha$ forest transmission (e.g. D'Aloisio et al. 2015). This correlation between gas

8 The exact value depends on I-front speeds, and to a more minor extent, the spectral index of the ionizing background. However, the uncertainty and scatter in this value should be smaller than the scatter resulting from different reionization times (e.g. D'Aloisio et al. 2019, Davies et al. 2019 Zeng \& Hirata 2020). temperature and Ly $\alpha$ transmission is, however, opposite of what is inferred from ULAS J0148+0600 based on galaxy counts (Becker et al. 2018, Kashino et al. 2020), suggesting that opacity fluctuations are not dominated by the temperature field for this one sightline.

\subsection{Building physical intuition from examples of forward-modelled data}

Using the IGM properties described in the previous sections, we compute the corresponding Ly $\alpha$ optical depth in each simulation cell. As we are interested in large-scale effects and use relatively low resolution simulations, we adopt the fluctuating Gunn-Peterson approximation (FGPA ${ }^{9}$ Gunn \& Peterson 1965. Rauch 1998, Weinberg \& et al. 1999) and estimate the optical depth by

$\tau_{\alpha}=f_{\text {rescale }} \times \sqrt{\frac{3 \pi \sigma_{\mathrm{T}}}{8}} c f_{\alpha} \lambda_{\alpha} H^{-1} n_{\mathrm{H}} x_{\mathrm{HI}}$

where $\sigma_{\mathrm{T}}, f_{\alpha}=0.416$ and $\lambda_{\alpha}=1216 \AA$ are the Thomson crosssection, oscillator strength, and Ly $\alpha$ rest-frame wavelength.

To better match observations, numerical simulations usually re-scale the optical depth by a constant factor within a given redshift window (either implicitly or explicitly; e.g. Chardin et al. 2017, D'Aloisio et al. 2018, Keating et al. 2018 Bosman et al. 2018), such that the modelled mean Ly $\alpha$ transmission agrees with the measured values: $\tau_{\alpha}\left(\lambda_{\text {obs }}\right) \rightarrow$ $\tau_{\alpha}\left(\lambda_{\text {obs }}\right) \times f_{\text {rescale }}$. This adds additional flexibility to the modelling (e.g. allowing for an arbitrary normalization and redshift evolution of the UV photoionization rate), ameliorates errors in the continuum subtraction, and/or compensates for modelling errors. However, such re-scaling wastes the predictive power of the model's emissivity and its redshift evolution. We further explain how we implement this rescaling factor in Section 4

Below we showcase our procedure for generating mock Ly $\alpha$ forest data. As a specific illustrative example, we use the maximum likelihood model in the forest posterior (presented in Section 5). This model is able to reproduce observations of the high-redshift galaxy UV LFs (Bouwens et al. 2015, Oesch et al. 2018), the CMB optical depth (Planck Collaboration et al. 2020), and the Ly $\alpha$ forest (Bosman et al. 2018). It corresponds to the following astrophysical model parameters $-\left(f_{*, 10}, \alpha_{*}, f_{\mathrm{esc}, 10}, \alpha_{\mathrm{esc}}, M_{\mathrm{turn}}, t_{*}\right)=(0.0448,0.488,0.0914$, $\left.-0.298,7.16 \times 10^{8} \mathrm{M}_{\odot}, 0.378\right)$ as well as $f_{\text {rescale }}=0.9+0.2 \times$ $(z-5.7)$ for flux normalization (see more in Section 4 ).

\subsubsection{Lightcones and cross correlation coefficients}

In Fig. 1, we show lightcone slices (with a thickness of 1.95 $\mathrm{Mpc}$ ) through the following fields: (i) density $(1+\delta)$, (ii) large-scale photoionization rate $\left(\Gamma_{\text {ion }}\right)$, (iii) gas temperature $\left(T_{\mathrm{g}}\right)$, (iv) the order unity fluctuations in the neutral hydrogen fraction from the $\operatorname{EoR}\left(x_{\mathrm{HI}}\right),(\mathrm{v})$ the residual neutral hydrogen fraction inside the ionized IGM, and (vi) the Ly $\alpha$ transmission $\left[F \equiv \exp \left(-\tau_{\alpha}\right)\right]$. In addition, to quantify how

9 In Appendix A we use high-resolution hydrodynamic simulations to quantify the error in modelling the large-scale Ly $\alpha$ transition caused by the FGPA. We include the resulting error covariance in our likelihood calculation, as detailed below. 


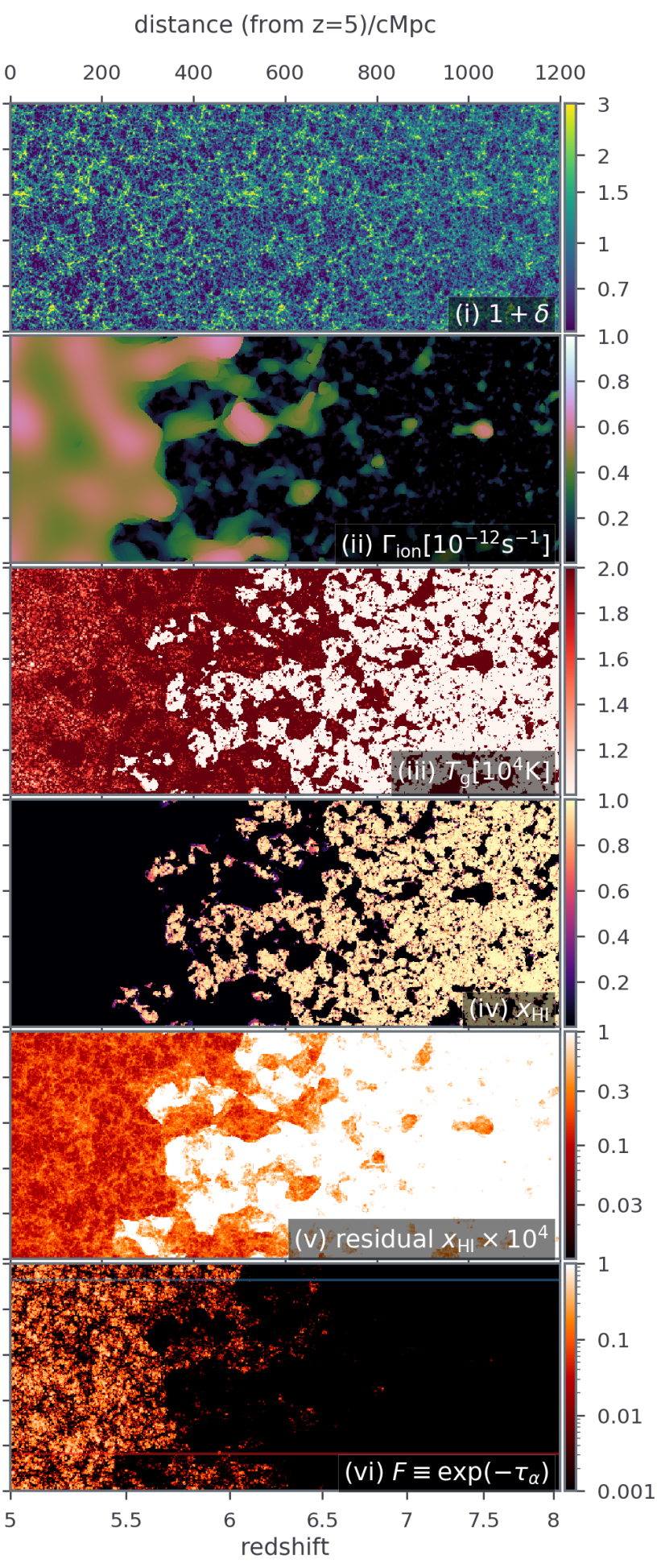

Figure 1. 2D slices through lightcones with a spatial scale of $500 \mathrm{cMpc}$ on the vertical axis and a thickness of $1.95 \mathrm{Mpc}$, spanning a redshift range of $5<z \lesssim 8$ (corresponding to a $1.2 \mathrm{cGpc}$ sight length) for the reference model (i.e. the maximum likelihood model in the forest posterior in Fig. 5). From top to bottom, the panels correspond to: (i) overdensity $(1+\delta)$; (ii) locally averaged UVB ( $\Gamma_{\text {ion }}$ in units of $10^{-12} \mathrm{~s}^{-1}$ ); (iii) temperature $\left(T_{\mathrm{g}}\right)$; (iv) neutral hydrogen fraction $\left(x_{\mathrm{HI}}\right.$ on a linear scale between 0 and 1$)$; $(\mathrm{v})$ residual neutral hydrogen fraction $\left(x_{\mathrm{HI}}\right.$ on a logarithmic scale between $10^{-6}$ and $\left.10^{-4}\right)$; and (vi) Ly $\alpha$ transmission $\left(F \equiv e^{-\tau_{\alpha}}\right)$. The red and blue lines in the bottom panel mark the two sample sightlines shown in Fig. 3

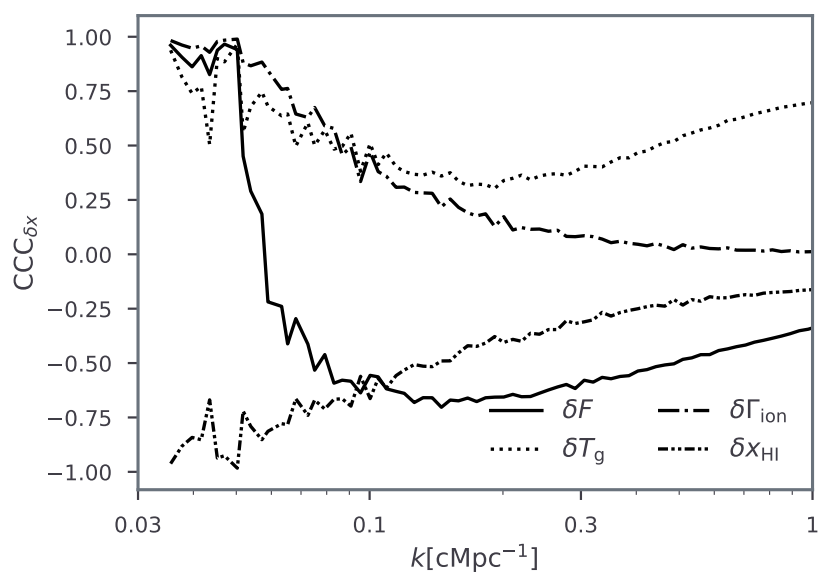

Figure 2. Cross correlation coefficients between the overdensity field $(\delta)$ and: (i) temperature $\left(T_{\mathrm{g}}\right.$ ), (ii) photonionization rate $\left(\Gamma_{\text {ion }}\right)$, (iii) neutral hydrogen fraction $\left(x_{\mathrm{HI}}\right)$, (iv) Ly $\alpha$ transmission $(F)$, for the reference model at $z=5.8\left(\bar{x}_{\mathrm{HI}} \sim 0.14\right)$.

these fields correlate with the underlying density on various scales, we calculate the cross correlation coefficient (CCC) defined as the cross-correlation power between density and a field, $P_{\delta \mathrm{x}}$, normalized by their auto-correlation power: $\mathrm{CCC}_{\delta \mathrm{x}}(k)=P_{\delta \mathrm{x}}(k) /\left(\sqrt{P_{\delta \delta}(k)} \sqrt{P_{\mathrm{xx}}(k)}\right)$. Fig. 2 shows CCCs between $F, T_{\mathrm{g}}, \Gamma_{\text {ion }}, x_{\mathrm{HI}}$ and $\delta$ using a snapshot at $z \sim 5.8$ (see projections in Fig. 6). We summarize some key trends below.

(i) The underlying density (panel i) plays a key role in determining the UV ionizing background (panel ii) and the reionization morphology (panel iv). Regions near high-density peaks become ionized first with the His bubbles spreading into the large-scale voids at later times (i.e. "inside-out" reionization; Furlanetto et al. 2004, Iliev et al. 2006, Lee et al. 2008, Choudhury et al. 2009||Friedrich et al. 2011; Bauer et al. 2015; Mesinger 2016; Hutter et al. 2017) and overlapping with each other to complete the EoR (at $z \sim 5.5$ in this model). This can also be seen in the CCCs: $x_{\mathrm{HI}}$ anti-correlates with $\delta$ and this anti-correlation strengthens towards smaller $k$ (larger scales). A similar trend is also found for the photoionization rate which correlates with density. The characteristic scales of these correlations depend on the model (i.e. the luminosity-weighted galaxy bias) and stage of EoR (e.g. McQuinn et al. 2007, Zahn et al. 2011).

(ii) The IGM temperature (panel iii) shows both largescale and small-scale structure. Large-scale hot regions are evident in the temperature maps down to $z \sim 5.2$, after the end of the EoR in this model. These trace the largescale underdensities that were the last to reionize (panel iv). However, large-scale structure is not evident at lower redshifts, with the IGM cooling towards $T_{\lim }(1+\delta)^{1 / \gamma}$ (see equation 8). Indeed, from Fig. 2 we confirm that $\mathrm{CCC}_{\delta T_{\mathrm{g}}}$ at $z=5.8$ has a non-monotonic relation with $k$, which rises towards scales smaller than $k \sim 0.2 \mathrm{cMpc}^{-1}$. Smaller scales are dominated by the temperature-density relation, while larger scales are dominated by EoR morphology.

(iii) The residual neutral hydrogen fraction (panel v) has a complex, multi-scale structure as it depends on the density, ionizing background and temperature (see equation 4). On large-scales ( $\gtrsim 50 \mathrm{cMpc})$, the residual $x_{\mathrm{HI}}$ anti-correlates with the $\Gamma_{\text {ion }}$ field, while on small-scales it can be seen to 


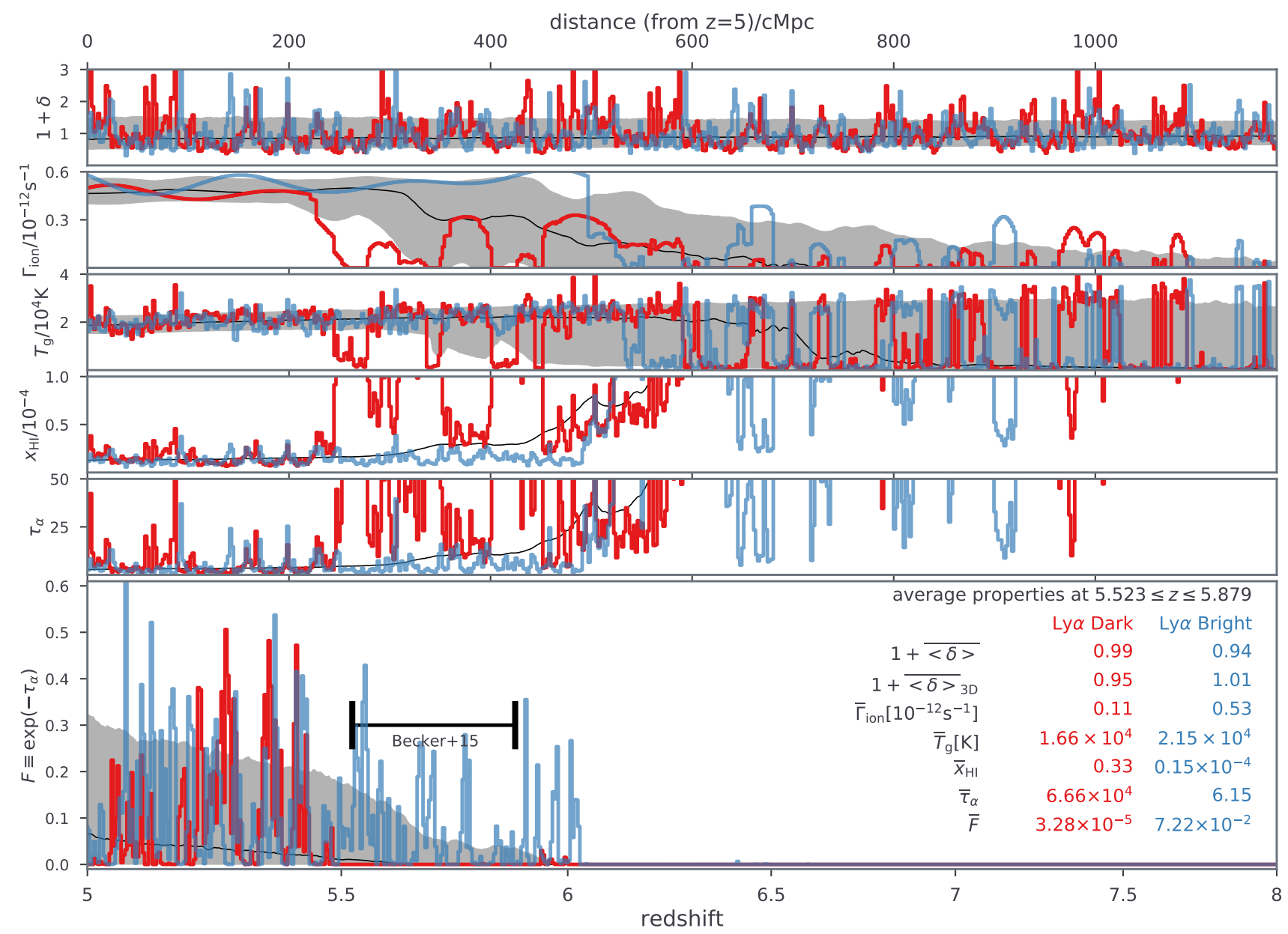

Figure 3. From top to bottom: overdensity $(1+\delta)$, locally averaged UVB $\left(\Gamma_{\text {ion }}\right)$, gas temperature $\left(T_{\mathrm{g}}\right)$, residual neutral hydrogen

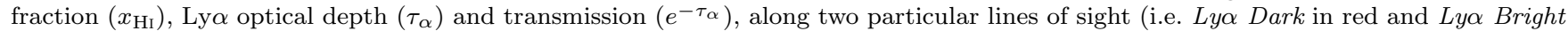
in blue; these two sightlines are also marked by the two horizontal lines in panel (v) of Fig. 1). The sightlines are shown with thick colored curves while the median and $[14,86]$ percentiles of the entire $3 \mathrm{D}$ lightcone are presented as the thin black lines and shaded regions. The average properties along the sightline at $5.523 \leqslant z \leqslant 5.879$ (the redshift range of the $110 h^{-1} \mathrm{cMpc}$ Ly $\alpha$ GP trough from Becker et al. 2015 ) are listed in the bottom right corner. We also show the density averaged over a $3 \mathrm{D}$ volume corresponding to $50 \times 50 \times 110 h^{-3} \mathrm{cMpc}^{3}$,

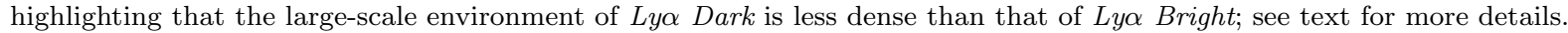

correlate/anti-correlate with the density/temperature fields (see also D'Aloisio et al. 2015. Davies \& Furlanetto 2016 D'Aloisio et al. 2018; Keating et al. 2018).

(iv) The Ly $\alpha$ optical depth (see the transmission in panel vi) generally decreases towards lower redshifts with fluctuations following the density field (panel i) and the inferred residual Hi fraction (panel v; see equation 9). We can clearly see long patches with $\operatorname{Ly} \alpha$ transmission lower than a thousandth on scales larger than $10 \mathrm{cMpc}$ post reionization with some even surviving at $z \sim 5$. We see that the transmitted flux and density anti-correlate on scales smaller than $k \gtrsim 0.05 \mathrm{cMpc}^{-1}$ (i.e. $\lesssim 130 \mathrm{cMpc}$ ), with the strongest anti-correlation occurring around $k \sim 0.1 \mathrm{cMpc}^{-1}$ (i.e. $\sim 60 \mathrm{cMpc}$ ). On extremely large scales however Ly $\alpha$ transmission is tightly correlated with the underlying density through the ionizing background.

\subsubsection{Spectra}

In Fig. 3, we show two example 1D sightlines from this light-

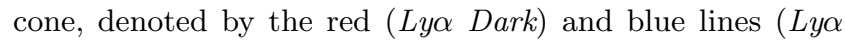

Bright) in panel (v) of Fig. 1. Ly 1 Dark was chosen as it exhibits a long, $110 h^{-1} \mathrm{cMpc}$ GP trough over the redshift interval $5.52 \leqslant z \leqslant 5.88$, consistent with ULAS J0148+0600 (Becker et al. 2015. see the bottom panel of Fig. 3). Ly $\alpha$ Dark shows an average transmission of $<10^{-4}$ over this red-

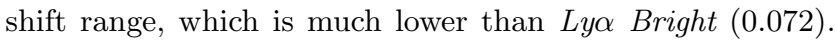
The latter corresponds to a more typical sightline at these redshift 10

The panels in the figure correspond to the matter overdensity, ionizing background, temperature, neutral fraction, optical depth and Ly $\alpha$ transmission, from top to bottom. We note that this ML model resulted in a rescaling factor very close to unity $\left(f_{\text {rescale }}=0.86-0.96\right.$; Section 4). Thus, the theoretical spectra required only minor calibration using this hyperparameter, in order to be consistent with the observed data. We also list the mean values over the red-

10 Among all $256^{2}=65536$ lines of sight in this maximum likelihood model, we find only 179 with a mean transmission lower than 0.001 . This is broadly consistent with current observations (1 out of $\sim 300 ;$ Bosman 2020). 

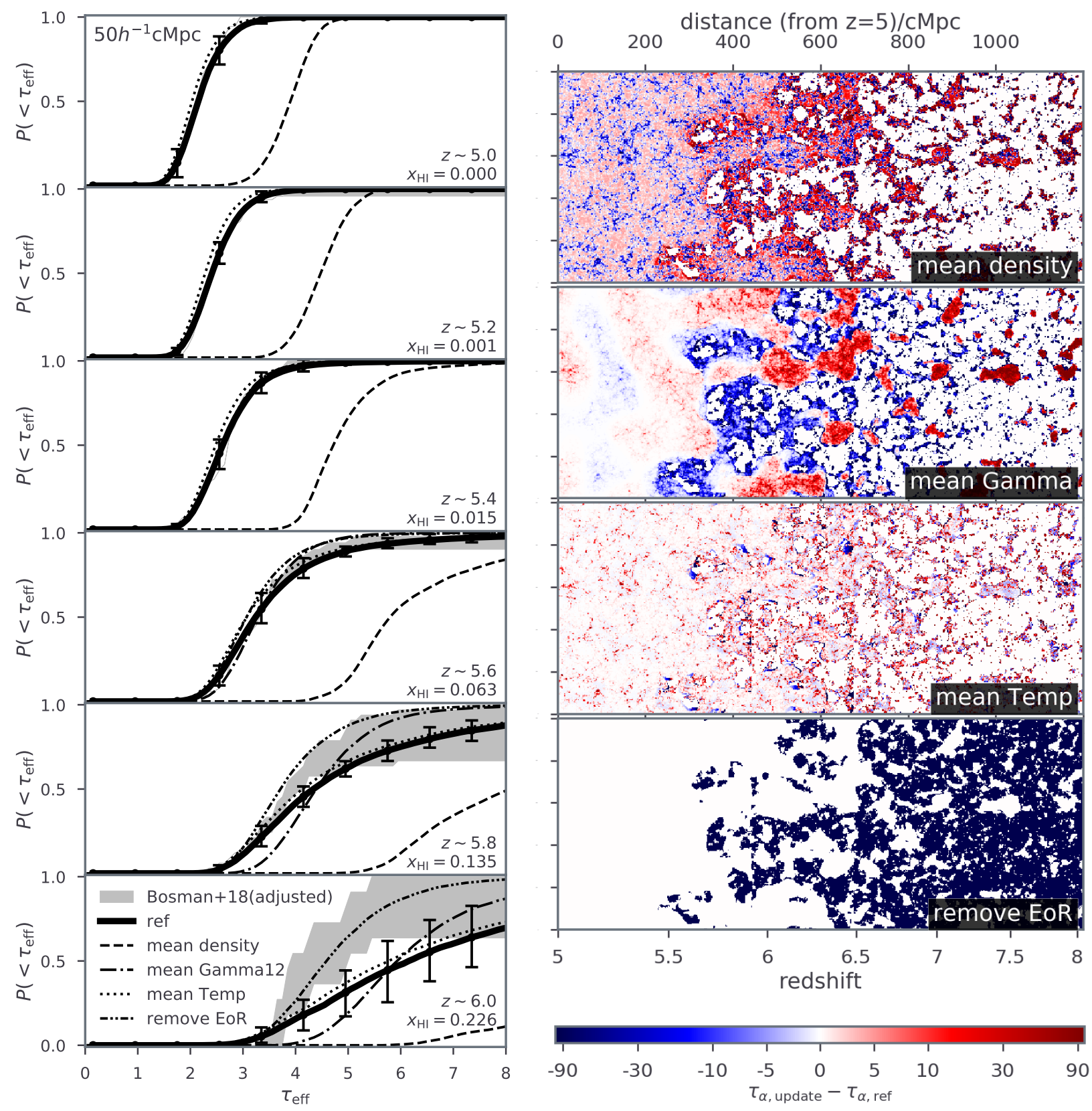

Figure 4. Left panels: Mean CDFs of $\tau_{\text {eff }}$ averaged over $50 h^{-1} \mathrm{cMpc}$ at $z=5-6$ from our maximum likelihood parameter combination (ref) are shown with thick black lines, with cosmic variance uncertainties $([14,86]$ percentiles) indicated for a subset of the bins. We also show CDFs resulting from removing the spatial fluctuations in various component fields (see text for details). The gray shaded regions span the observational estimates (see Sec. 33. Right panels: lightcone slices illustrating the change in the Ly $\alpha$ optical depth relative to the reference model for each simulation that removes fluctuations in the indicated field. Note that the colorbar is linear between 0 and \pm 10 and logarithmic from \pm 10 to \pm 100 . These slices illustrate that large-scale fluctuations in this model are predominantly driven by a patchy EoR and a patchy UVB.

shift interval $5.52 \leqslant z \leqslant 5.88$ in the bottom panel. We see that on average Ly $\alpha$ Dark has a lower ionization rate as well a lower temperature while the average densities along the two slightlines are both close to the cosmic mean.

We see that the different levels of Ly $\alpha$ transmission between these two sightlines are mostly driven by the neutral hydrogen and UVB fluctuations. In the region corresponding to the long GP trough, Ly $\alpha$ Dark pierces through a few remaining cosmic Hi patches (with $\Gamma_{\text {ion }} \sim 0$ ). Although these
Hi patches combined span less than half of the GP through length, we see from the $\Gamma_{\text {ion }}$ panel that the ionized IGM between the Hi patches is exposed to a below-average UVB (see also Keating et al. 2020b, Nasir \& D'Aloisio 2020). As discussed above, both fields correlate with the underlying density on large scales: the regions last to ionize and those with a small UVB both correspond to large-scale underdensities with comparably few star-forming galaxies. This is quantitatively evident when we compute the volume averaged 
Qin et al.

density around the sightlines, showing that Ly $\alpha$ Dark lies in a large-scale underdensity, $1+\bar{\delta}_{3 \mathrm{D}}=0.95$ when average over a volume of $50 \times 50 \times 110\left(h^{-1} \mathrm{Mpc}\right)^{3}$. This picture is also consistent with follow-up observations of J0148+0600 that show a dearth of galaxies at the location of its GP trough (Becker et al. 2018; Kashino et al. 2020).

\subsubsection{Fields that determine Lya fluctuations}

Using our maximum likelihood (reference) parameter set, we now further quantify which fields are most relevant for largescale opacity fluctuations. In the left panels of Fig. 4 we show the cumulative probability distributions (CDFs) of the effective optical depth defined as $\tau_{\text {eff }} \equiv-\ln \left[\left\langle\exp \left(-\tau_{\alpha}\right)\right\rangle_{\mathrm{L}}\right]$, where we chose $L=50 \mathrm{~h}^{-1} \mathrm{cMpc}$ in order to facilitate comparison against published observations (shown as gray shaded regions and further discussed in Section 3. Thick, solid curves correspond to the mean CDFs from our ML model, for redshift bins spanning $z=5-6{ }^{11}$ The other curves in these panels were constructed by removing fluctuations in a single component field, and then recomputing the resulting optical depths (though keeping the same normalization factor, $\left.f_{\text {rescale }}\right)$. Specifically, we replace the density (mean density), photo-ionization rate (mean Gamma) and temperature ( mean Temp) with their ionized volume averaged values at each redshift. For remove EoR, we remove all cosmic HI patches by assigning to them the mean values of the photoionization rate and temperature in the ionized regions, and then recomputing $\tau_{\alpha}$ in each cell.

In the right panels, we also show the lightcone visualizations of the corresponding change in the optical depth with respect to the reference $\mathrm{ML}$ model (see the transmission in Fig. 1). As expected, ignoring density fluctuations results in a large shift in the CDFs, but does not dramatically impact their shapes - there is only a minor decrease in the abundance of high $\tau_{\text {eff }}$ regions. Thus density fluctuations alone cannot efficiently generate long GP troughs (Becker et al. 2015)). Ignoring the temperature fluctuations has a minor impact, mostly on small scales.

The most striking difference comes from the patchy EoR and UVB fields. We see that the fluctuations in both fields have a relatively large impact on the $\tau_{\text {eff }}$ CDFs during the final stages of the $\operatorname{EoR}(z \gtrsim 5.5)$, affecting the opacity on large scales. In agreement with the discussion in the previous section (see also e.g. Keating et al. 2020b. Nasir \& D'Aloisio 2020), their impact is additive - they both widen the CDFs, extending the high-value tails of $\tau_{\text {eff }}$. Post EoR as the mean free path increases, the fluctuations in the UVB become negligible when computing the $\tau_{\text {eff }} \mathrm{CDF}^{12}$

11 The ML model seems to be in (very) mild tension with the data at $z=6$. We note however that preliminary, updated data from the XQR30 large VLT program (PI: V. D'Odorico) are in better agreement with this reference model at $z=6$.

12 We caution that our model does not include AGN. AGNs are expected to dominate the UVB at lower redshifts, $z \lesssim 3-4$ (e.g. Madau \& Haardt 2015, Mitra et al. 2015 Qin et al. 2017. Kulkarni et al. 2019); however, some works have evoked rare, bright AGN to explain the observed large scale $\tau_{\text {eff }}$ fluctuations at $z \sim 5-6$ (e.g. Chardin et al. 2017, Meiksin 2020). Regardless, our models are able to fit the data at $z>5$ without AGN (see also, e.g. Nasir \& D'Aloisio 2020 Keating et al. 2020b.

\section{OBSERVATIONAL DATA}

We constrain our model using the most up to date, public Ly $\alpha$ forest sample ${ }^{13}$ from Bosman et al. (2018). Specifically, we use the "SILVER" subsample of 51 QSO that have high quality spectra with signal-to-noise ratio $(\mathrm{S} / \mathrm{N}) \geqslant 5.3$ in the continuum. The spectra are binned over $50 h^{-1} \mathrm{cMpc}$, resulting in 217 flux measurements over the redshift interval $4.9 \leqslant z \leqslant 6.1$ (i.e. $\langle F\rangle_{50 h^{-1} \mathrm{cMpc}}$ with uncertainties of $\left.\delta_{\langle F\rangle_{50 h-1}{ }_{\mathrm{cMpc}}}\right)$. We then have the following number of samples, $N_{\text {sample }}=18,47,57,51,33$ and 11 at $z=5.0,5.2,5.4$, $5.6,5.8$ and 6.0 , respectively. The effective optical depth is then calculated as $\tau_{\text {eff }}=-\ln \langle F\rangle_{50 h^{-1} \mathrm{cMpc}}$ and, for nondetections, $\tau_{\text {eff }} \geqslant-\ln \left(2 \delta_{\langle F\rangle_{50 h}-1 \mathrm{cMpc}}\right)$ as the lower limit 14

We then rescale these effective optical depths to account for the improved continuum reconstruction from Bosman et al. (2020). Specifically, we use the PCAnominal method from Bosman et al. (2020), scaling the transmission by the ratio of the two continuum estimates, $\tau_{\text {eff }}=-\ln \left(\langle F\rangle_{50 h^{-1} \mathrm{cMpc}} \mathscr{R}\right)$, and the lower limits to $\tau_{\text {eff }} \geqslant-\ln \left(2 \delta_{\langle F\rangle_{50 h}-1_{\mathrm{cMpc}}} \mathscr{R}\right)$ for non-detections. Here, $\mathscr{R} \equiv$ $\langle F\rangle_{\text {obs }}^{\text {Bosman }+20} /\langle F\rangle_{\text {obs }}^{\text {Bosman }+18}$ represents the mean flux ratio between Bosman et al. (2018) and Bosman et al. (2020), which is $0.93,0.87,0.91,0.87,1.03$ and 1.60 at $z=5.0,5.2$, $5.4,5.6,5.8$ and 6.0, respectively. We note that this only has a noticeable change to the CDF of $\tau_{\text {eff }}$ at $z=6$ where the peak is at a slightly lower value.

The resulting observed CDFs of $\tau_{\text {eff }}$ are shown as the gray shaded regions in Fig. 4 When performing EoR inference from these observational estimates, we consider flat likelihoods between the two extremes (i.e. the gray shaded region; see more in Section 5.1.

\section{SUMMARY OF MODEL PARAMETERS}

Before proceeding to our MCMC results, we summarize the free parameters of our model and the associated prior ranges. For computational convenience in this proof-of-concept work we restrict our parameter exploration to the most relevant astrophysical parameters (i.e. responsible for the largest variation in the forward-modelled data); for a complete list of parameters see Section 2 and Park et al. (2019). We also do not co-vary cosmological parameters, keeping the same underlying density field. In future work, we will relax this assumption and quantify joint constraints on astrophysical and cosmological parameters from the Ly $\alpha$ forest.

Our model consists of six astrophysical parameters characterizing the UV emission of galaxies:

(i) $f_{*, 10}$, the fraction of galactic baryons inside stars, defined for galaxies residing in halos with $M_{\text {vir }}=10^{10} \mathrm{M}_{\odot}$. We vary $f_{*, 10}$ between $10^{-3}$ and 1 with a flat prior in log space;

(ii) $\alpha_{*}$, the power-law index of the stellar fraction to halo mass relation. We vary it between -0.5 and 1 with a flat

13 www.sarahbosman.co.uk/research

14 Note that a few objects in Bosman et al. (2018) have different lower limits from $\tau_{\text {eff }} \geqslant-\ln \left(2 \delta_{\langle F\rangle_{50 h}-1 \mathrm{cMpc}}\right)$ as they adopt the peak limit definition proposed by Becker et al. (2015). 
prior, noting that high-redshift observations of galaxy UV LFs suggest $\alpha_{*} \sim 0.5$.

(iii) $f_{\text {esc,10, }}$, the UV ionizing escape fraction, defined for galaxies residing in halos with $M_{\mathrm{vir}}=10^{10} \mathrm{M}_{\odot}$. Similarly to $f_{*, 10}$, we vary $f_{\text {esc }, 10}$ between $10^{-3}$ and 1 with a flat prior in log space;

(iv) $\alpha_{\text {esc }}$, the power-law index of the ionizing escape fraction to halo mass relation. Although poorly known, some works suggest increasing or constant escape fractions towards lower mass galaxies (Ferrara \& Loeb 2013, Kimm \& Cen 2014, Paardekooper et al. |2015, Xu et al.| 2016). We thus have a wider prior on the negative range, varying $\alpha_{\text {esc }}$ between -1 and 0.5 (with a flat prior);

(v) $M_{\text {turn }}$, the turnover halo mass below which the number density of halos hosting star-forming galaxies become exponentially suppressed. We vary $M_{\text {turn }}$ between $10^{8}$ and $10^{10} \mathrm{M}_{\odot}$ with a flat prior in $\log$ space. The former corresponds to the atomic cooling threshold while the latter corresponds to current $H S T$ sensitivity limits beyond which we see a high occupancy fraction of star forming galaxies; and

(vi) $t_{*}$, the star-formation timescale as a fraction of the Hubble time. We vary $t_{*}$ between 0 and 1 with a flat prior.

We also introduce two hyper-parameters to describe the optical depth normalization factor, $f_{\text {rescale }}$ from equation (9). Hyper (or nuisance) parameters can characterize systematics and errors, and are marginalized over to obtain the final constraints on the desired parameters. As mentioned in the associated discussion, the $f_{\text {rescale }}$ normalization factor can account for errors in the continuum subtraction as well as modelling errors. However, it comes with the cost of losing the intrinsic predictive power of the model. In order to preserve some of the predictive power of our model, we assign these hyper-parameters a prior centered around $f_{\text {rescale }}=1$ (with unity corresponding to no calibration). The chosen widths are fairly arbitrary, with the fiducial Park et al. (2019) model included within $1 \sigma$; however, below we also explore an extreme case that essentially allows infinitely wide priors. Specifically, the two hyper-parameters of our fiducial MCMC are:

(vii) $f_{\text {rescale }}(z=5.7)$, the rescaling factor at $z=5.7$, sampled in $\log$ space between $10^{-0.5}$ and $10^{0.5}$ with a Gaussian prior of a zero mean (i.e. $\left\langle\log _{10} f_{\text {rescale }}(z=5.7)\right\rangle=0$ ) and a width of $\sigma \log _{10} f_{\text {rescale }}(z=5.7)=1.5$;

(viii) $\mathrm{d} f_{\text {rescale }} / \mathrm{d} z$, the slope of the rescaling factor as a function of redshift, sampled between -1.5 and 1.5 with a Gaussian prior of a zero mean (i.e. $\left\langle\mathrm{d} f_{\text {rescale }} / \mathrm{d} z\right\rangle=0$ ) and a width of $\sigma \mathrm{d} f_{\text {rescale }} / \mathrm{d} z=2.0$.

\section{INFERRING GALAXY AND IGM PROPERTIES FROM THE Ly $\alpha$ FOREST}

In this section, we use the 21CMMC sampler (Greig \& Mesinger 2015, 2017, 2018) to perform three MCMC runs, quantifying EoR and galaxy constraints with and without the Ly $\alpha$ forest data. For computational efficiency, our MCMCs are done on smaller volumes (250 cMpc on a side) with the same cell size as the example shown in Section 2 (i.e. cell lengths of $250 / 128=1.95 \mathrm{cMpc}$ ). Our MCMC runs typically require $\sim 10^{5}$ samples to reach convergence, which take $\sim 15000 \mathrm{CPU}$ hours.

\subsection{Computing the likelihood}

For a given combination of galaxy and hyperparameters, $\theta$, we compute the associated likelihood from Ly $\alpha$ forest fluctuations, $\mathscr{L}_{\alpha}(\theta)$, according to the following:

(i) We forward model the corresponding 3D lightcone of the Ly $\alpha$ optical depth as described in Section 2.3

(ii) At a given redshift $z$, we compute the $\mathrm{PDF}^{15}$ of the effective optical depths from a sample of $N_{\text {sample maximally- }}$ separated (to minimize spatial coherence) sightlines, with $N_{\text {sample }}$ corresponding to the sample size of the observational data at $z$ as discussed in Sec. 3 .

(iii) We repeat step (ii) $N_{\text {realization }}=150$ times, ensuring each sightline in the lightcone is not selected more than once. From these realizations we compute the mean PDF $\left(\phi_{\text {model }}\right)$, and the cosmic variance error matrix $\left(\Sigma_{\mathrm{CV}}\right.$; see more in Appendix B).

(iv) We calculate the total error covariance matrix ${ }^{16}$ as $\Sigma=\Sigma_{\mathrm{CV}}+\Sigma_{\mathrm{GP}}$, where $\Sigma_{\mathrm{GP}}$ corresponds to the error from the FGPA (see Appendix A.

(v) We calculate the difference ${ }^{17}$ between the modelled mean PDF and the observed PDF, $X$.

(vi) From (iv) and (v) we compute a $\chi^{2}$ likelihood for this redshift, according to $\ln \mathscr{L}_{z}(\theta)=-0.5 X^{T} \Sigma^{-1} X$.

(vii) We repeat steps (ii) to (vi) for all redshift bins used in our analysi: ${ }^{18}(z=5.4,5.6,5.8$ and 6.0), summing up the log likelihoods: $\ln \mathscr{L}_{\alpha}(\theta)=\sum_{z} \ln \mathscr{L}_{z}(\theta)$.

Finally, we obtain the total likelihood with: $\mathscr{L}(\theta)=$ $\mathscr{L}_{\alpha} \times \mathscr{L}_{\mathrm{LFs}} \times \mathscr{L}_{\mathrm{DF}} \times \mathscr{L}_{\tau_{e}}$, where the final three terms correspond to current, robust EoR constraints from: (i) the galaxy UV LFs at $z=6-10$ from Bouwens et al. (2015, 2016) and Oesch et al. (2018); (ii) the upper limit on the neutral hydrogen fraction at $z \sim 5.9, x_{\mathrm{HI}}<0.06+0.05(1 \sigma)$, measured using the dark fraction of quasar spectra (McGreer et al. 2015); and (iii) the Thomson scattering optical depth of CMB photons reported by Planck Collaboration et al. $(2020), \tau_{e}=0.0561 \pm 0.0071(1 \sigma)$; respectively. For more details about these observations and the functional forms of the corresponding likelihoods, see Park et al. (2019).

15 As noted by Choudhury et al. 2021), we use PDFs instead of the more commonly presented CDFs when computing the likelihood, as CDFs have stronger covariances between bins.

16 We do not include flux uncertainties in the total covariance matrix, as we verify they are far subdominant compared to the FGPA and cosmic variance errors for this observational sample. In future work, we will extend our framework and forward model each individual sightline, including the corresponding flux errors. 17 As mentioned in Section 3. we consider flat likelihoods between the two extreme cases shown in Fig. 4 For instance, assuming the observed number density in a particular $\tau_{\text {eff }}$ bin has the limits $\phi_{\text {obs, upper }}$ and $\phi_{\text {obs,lower }}$, we take $X=0$ when $\phi_{\text {obs,lower }} \leqslant \phi_{\text {model }} \leqslant \phi_{\text {obs, upper }}$, and $X=\min \left(\left|\phi_{\text {model }}-\phi_{\text {obs, upper }}\right|,\left|\phi_{\text {model }}-\phi_{\text {obs,lower }}\right|\right)$ otherwise.

18 We expect our models to become less accurate at lower redshifts since, (i) we do not account for the contribution of AGN to the UV background; and (ii) we do not capture the spatial clustering of biased absorbers such as DLAs. As discussed above, we expect these approximations to be reasonable at $z>5$; however for this proof-of-concept work, we conservatively restrict ourselves to the highest redshift bins that are the most sensitive to the EoR. In future works, we will explore extending this range, including an additional population of bright AGN. 


\subsection{Posteriors}

Fig. 5 presents the MCMC results including: the marginalized posterior distributions of the model parameters (the corner plot in the lower left), the EoR histories (panels a),

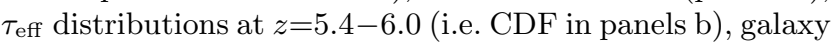
UV LFs at $z=6-15$ (panels c), the UVB evolution (panel d) as well as the PDF of the CMB Thomson scattering optical depth, $\tau_{e}$ (panel e).

We perform the following three MCMC runs:

- no_forest - does not use the $\tau_{\text {eff }}$ PDFs, with the likelihood corresponding to $\mathscr{L}(\theta)=\mathscr{L}_{\mathrm{LFs}} \times \mathscr{L}_{\mathrm{DF}} \times \mathscr{L}_{\tau_{e}}$. This run roughly corresponds to our current state of knowledge, before accounting for Ly $\alpha$ opacity fluctuations. Here, we vary the astrophysical parameters (i) - (vi) from Sec. 4.

- forest - additionally includes the observed $\tau_{\text {eff }}$ PDFs discussed in Sec. 3 thus $\mathscr{L}(\theta)=\mathscr{L}_{\alpha} \times \mathscr{L}_{\mathrm{LFs}} \times \mathscr{L}_{\mathrm{DF}} \times \mathscr{L}_{\tau_{e}}$. Here, we vary the astrophysical and nuisance parameters, (i) - (viii) from Sec. 4. This corresponds to our fiducial MCMC run.

- forest_fluc - unlike forest, does not sample the $f_{\text {rescale }}$ hyper-parameters from their priors. Instead we normalize each PDF by insuring the mean flux, $\left\langle\exp \left(-\tau_{\alpha}\right)\right\rangle$, matches the observed mean flux. This roughly corresponds to assuming infinitely wide priors for $f_{\text {rescale }}$. Therefore, this run only varies the astrophysical parameters (i) - (vi) from Sec. 4.

Below we discuss the posterior of each of these in turn.

\subsection{1 no_forest}

The no_forest posterior is shown with the orange, shaded regions. As noted by Park et al. (2019), current EoR observations already place constraints on some of our model parameters, even without making use of the $\tau_{\text {eff }}$ PDF data. In particular, galaxy LFs constrain the stellar to halo mass relation, parametrized here through $f_{*, 10} / t_{*}$ and $\alpha_{*}$, to within a factor of few (see also, e.g. Tacchella et al. 2018 Behroozi et al. 2019, Yung et al. 2019, Qin et al. 2020b). This is also evident from the tight recovery of the UV LFs at the bright end $\left(M_{\mathrm{UV}} \lesssim-15\right)$. However, we do not detect a faint-end turnover in the LFs, resulting from inefficient star formation in galaxies hosted by halos with masses less than $M_{\text {turn }}$; current UV LFs only provide upper limits on this parameter $\left(\lesssim 5 \times 10^{9} \mathrm{M}_{\odot} ;\right.$ e.g. Bouwens et al. 2015b). The ionizing escape fraction is only weakly constrained by the dark frac-

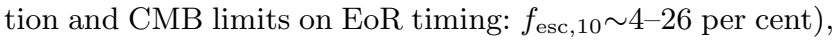
while its scaling with the mass of the host halo remains uninformed (see also e.g. Haardt \& Madau 2012, Kuhlen \& Faucher-Giguère 2012, Robertson et al. 2015, Price et al. 2016).

\subsection{2 forest}

Additionally including the $\tau_{\text {eff }}$ data has a dramatic impact on the posterior (shown with the red curves in Fig. 5). Most importantly, we note from panel (a1) that the forest data requires late reionization. The final overlap stages of the EoR, corresponding to when $>95 \%$ of the volume was ionized, occur at $z<5.6$. Our Bayesian framework provides statistical proof of previous suggestions that the EoR might have completed at $z<6$ (Lidz et al. 2007. Mesinger 2010, Kulkarni et al. 2019, Keating et al. 2020b Nasir \& D'Aloisio 2020). Our constraints on overlap at $z<5.6$ are also perfectly consistent with the recent, similar analysis by Choudhury et al. (2021), using their indirect parametrization of ionizing sources and IGM recombinations.

Moreover, we find that the $\tau_{\text {eff }}$ PDFs and the dark fraction upper limits are in mild tension $(\sim 1.5 \sigma$; see panel a 2$)$. The dark fraction tends to prefer earlier reionization, while the $\tau_{\text {eff }}$ PDFs require later reionization. Combining the two forces the marginalized posterior of EoR histories to have a very narrow tail bellow $z<6$ (see panel a1). We will revisit this mild tension in future work, using updated estimates of both data sets from the XQR30 large observational program (PI: V. D'Odorico).

As it helps nail down the timing of the EoR, the forest data dramatically improves constraints on the ionizing escape fraction. To be consistent with all of the data, reionization would need to end late (preferred by the broad $\tau_{\text {eff }}$ PDFs) but not too late or with an extended tail towards low redshifts (preferred by the modest values of $\tau_{e}$ and the dark fraction). These limit the ionizing contribution of both earlyforming, faint galaxies and their late-appearing, bright counterparts, resulting in $\alpha_{\text {esc }}$ that peaks around $-0.29_{-0.14}^{+0.29}$, a characteristic ionizing escape fraction of $f_{\mathrm{esc}, 10}=6.9_{-2.6}^{+3.8} \%$, as well as a weakly constrained $(\sim 1 \sigma)$ lower limit on $M_{\text {turn }}$ (i.e. $\gtrsim 2 \times 10^{8} \mathrm{M}_{\odot}$ ).

As we mentioned previously, in this initial study we do not enforce UV photon conservation, in favor of simulation speed (setting PHOTON_CONS=FALSE in $21 \mathrm{cmFASTV} 3$ ). This choice roughly leads to a +0.2 bias in the inferred $\alpha_{\text {esc }}$ parameter (Park et al. in prep). Taking this into account, we predict that the true marginalized posterior of $\alpha_{\mathrm{esc}}$ is consistent with zero and has a modest width of $\sigma_{\alpha_{\text {esc }}} \lesssim 0.3$. This suggests that the forest data disfavors a strong evolution of the ionizing escape fraction with the galaxy's halo (or stellar) mass (i.e. large $\left|\alpha_{\text {esc }}\right|$ ), consistent with recent results from hydrodynamical simulations of a handful high- $z$ galaxies in the relevant mass ranges (Kimm \& Cen 2014 Ma et al. 2015. Xu et al. 2016; Ma et al. 2020).

Finally, it is useful to point out a few sanity checks of our model. Firstly, we see that the recovered hyperparameters, accounting for systematic errors in continuum subtraction and theoretical modelling, are consistent with $f_{\text {rescale }}=1$. This implies that our intrinsic models of the forest can match the forest data, without significant "tuning". Secondly, we note from panel (d) that our forest posterior matches perfectly with estimates of the mean ionizing background at $z=5$ and 6 (denoted by black points with error bars; Bolton \& Haehnelt 2007b; Calverley et al. 2011, Wyithe \& Bolton 2011). It is worth noting that, although they are based on forest observations, we do not use these estimates of the UVB in our likelihood.

\subsection{3 forest_fluc}

The forest_fluc posterior is denoted with blue curves in Fig. 5. Recall that here we calibrate each forward modelled $\tau_{\text {eff }}$ PDF such that the mean flux matches the data, for every redshift bin and every parameter sample. This corresponds to an extreme case in which we have no idea how to put priors on $f_{\text {rescale. }}$ In effect, we are removing the "DC mode" 


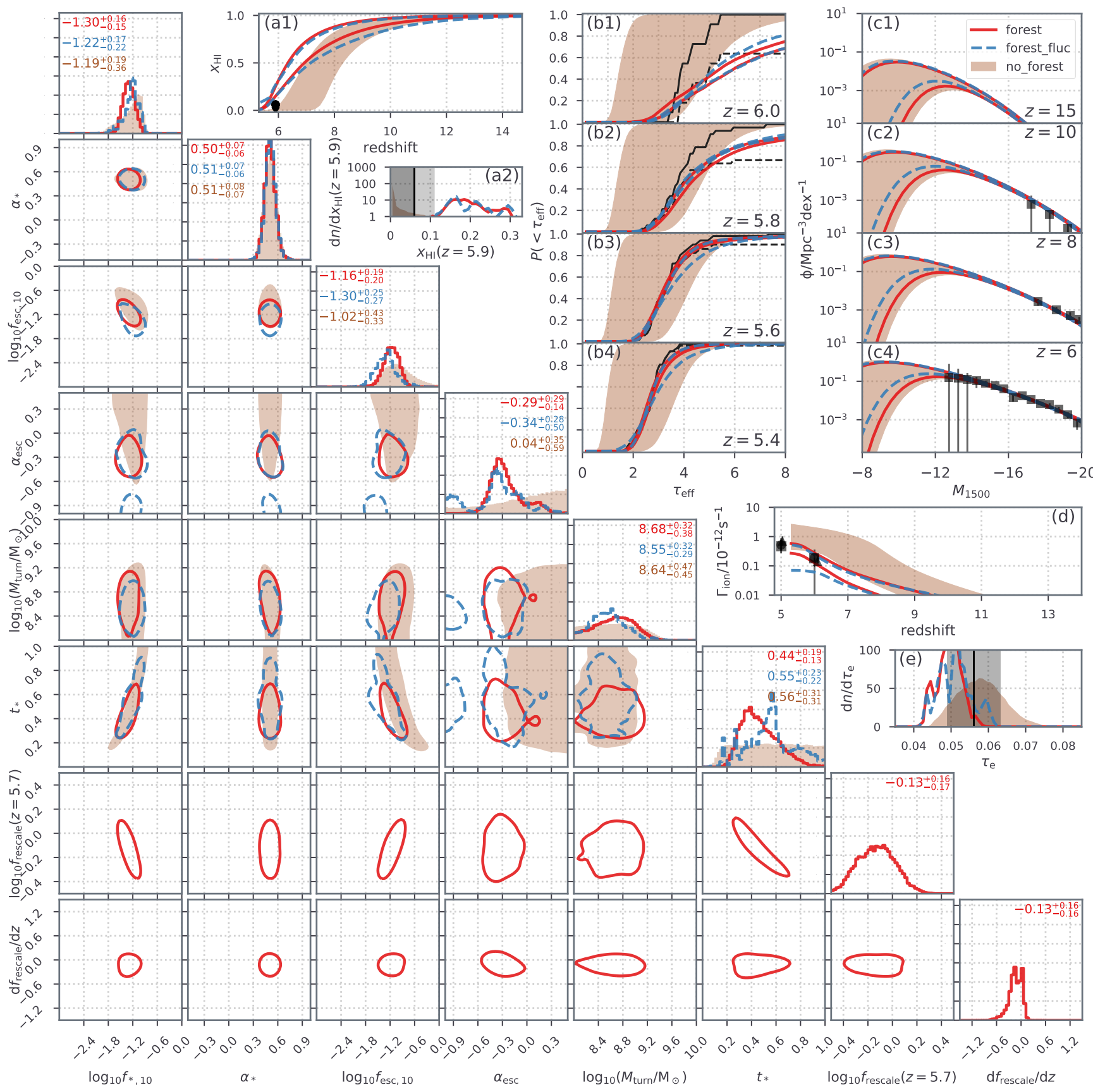

Figure 5. Marginalized posterior distributions of the model parameters with (forest; red solid lines \& forest_fluc; blue dashed lines) and without (no_forest; brown shaded areas) the forest data (Bosman et al.|2018). All three results use the observed galaxy LFs at $z=6-10$ (Bouwens et al. 2015, 2017 Oesch et al. 2018), upper limits on the neutral fraction at $z \sim 5.9$ from quasar spectra (McGreer et al. 2015), and Thomson scattering optical depth of the CMB (Planck Collaboration et al. 2020) as input constraints. The 2D distributions correspond to the 68 th percentiles. The medians with $[14,86]$ percentiles for each parameter are presented in the upper corner of the 1D PDFs (from top to bottom: forest, forest_fluc and no_forest, respectively). Note that all 1D PDFs have been normalized to have area (or integral) under the histogram equal to 1 . The upper right sub-panels present the median and [14, 86] percentiles of the neutral hydrogen ( $x_{\mathrm{HI}}$; panel a1); the CDFs of Ly $\alpha$ effective optical depths at $z=5.4-6.0$ (panels b1-b4); the UV LFs at $z=6-15$ (panels c1-c4); the evolution of the photoionization rate $\left(\Gamma_{\mathrm{ion}}\right.$; panel d); as well as the PDFs of $\tau_{e}$ (panel e) and $x_{\mathrm{HI}}$ at $z=5.9$ (panel a2) for the models presented in the posterior distributions. Observations including UV ionizing background measured by Bolton \& Haehnelt 2007b); Calverley et al. (2011); Wyithe \& Bolton 2011) are indicated in black.

of our forest models, mostly comparing the shapes of the PDFs over the observable range.

Even when calibrating to the observed mean flux, we recover the result that EoR must finish at $z<5.6$ to match the observed widths of the Ly $\alpha$ opacity distributions (panel a1). By comparing the blue curves to the red ones, we see that losing information on the mean flux expands the recovered posterior to include models with a very low ionizing background (see panel d). These are mostly sourced by small values of $\alpha_{\mathrm{esc}}$, corresponding to enhanced ionizing efficien- 

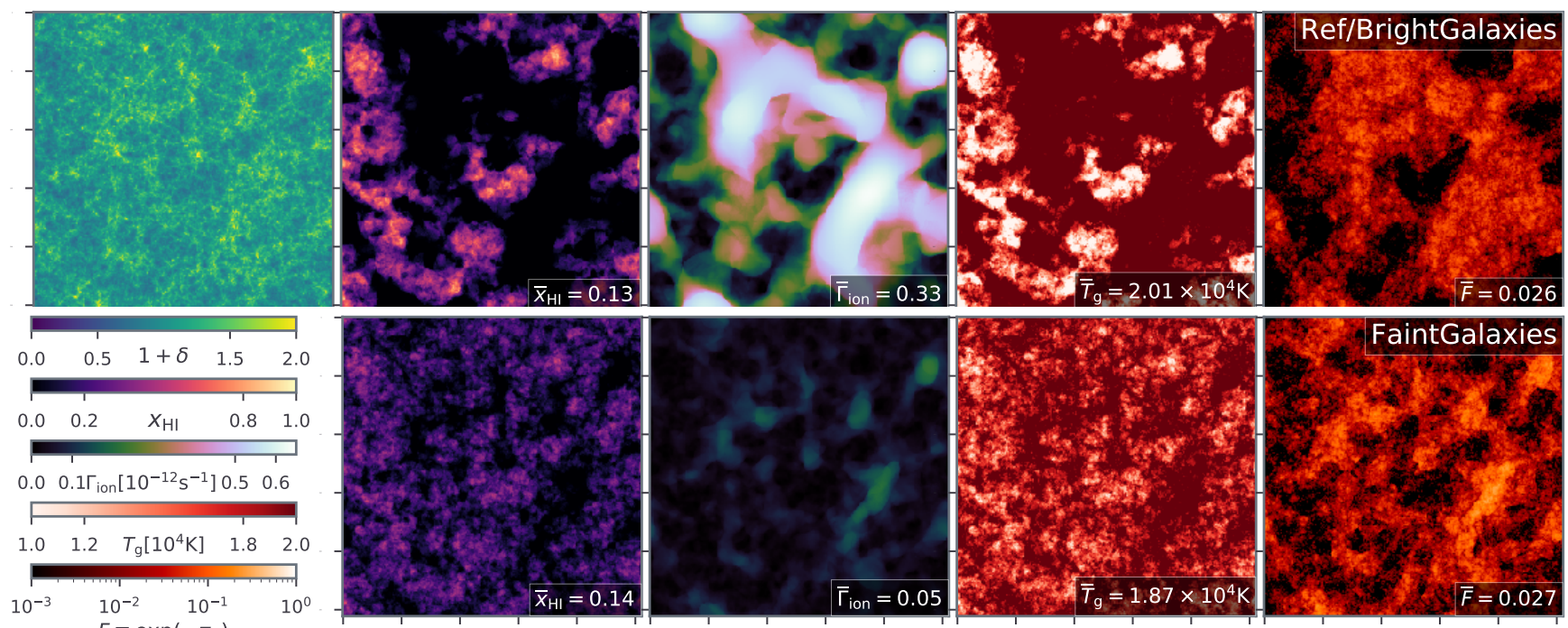

Figure 6. Slices (with a side length of $50 h^{-1} \mathrm{cMpc}$ and depth of $2 \mathrm{cMpc}$ ) of the density $(1+\delta)$, neutral hydrogen fraction $\left(x_{\mathrm{HI}}\right)$, photonionization rate $\left(\Gamma_{\mathrm{ion}}\right)$, gas temperature $\left(T_{\mathrm{g}}\right)$ and Ly $\alpha$ transmission flux from Ref/BrightGalaxies (upper subpanels) and FaintGalaxies (lower ones); see text for more details on these models. All slices correspond to $z=5.8$ where the two models have the same filling factor of $\mathrm{HI}$, and were rerun in larger volumes $(500 \mathrm{cMpc})$ compared to the MCMC for better visualization.

cies in galaxies hosted by low-mass halos. The corresponding EoR histories are also somewhat slower, since the fractional growth of less massive halos is slower than that of more massive ones. As a result, the marginal posterior on $\alpha_{\text {esc }}$ is bi-modal, with the smaller peak at low values being driven by a slightly better agreement with $\tau_{e}$ from Planck due to the more extended EoR.

\subsection{Can we distinguish between different reionization morphologies?}

In the previous section, we demonstrated that our models require reionization to be incomplete at $z<6$ in order to match the observed $\tau_{\text {eff }}$ PDFs, while in Sec. 2.3.3 we saw that both the patchy UVB and patchy EoR are needed to reproduce the longest GP troughs (see also Keating et al. $2020 \mathrm{~b})$. In this section, we check if the forest opacity fluctuations can distinguish between different reionization morphologies, at a fixed stage of the EoR. Constraining the bias of the dominant galaxy population through the EoR morphology would be immensely powerful, allowing us to distinguish between different galaxy models that result in similar reionization histories (e.g. McQuinn et al. 2007, Dixon et al. 2016. Mesinger et al. 2016 Ahn \& Shapiro 2020). Indeed, the ability to measure the morphology of the EoR and epoch of heating is one of the main reasons the cosmic $21-\mathrm{cm}$ signal will allow us to place $\sim$ percent level constraints on the properties of the unseen first galaxies (e.g. see the recent review in Mesinger 2019).

In Fig. 6] we show slices through the fields of two models at $z=5.8$, chosen to have the same neutral fraction $\left(\bar{x}_{\mathrm{HI}} \sim 0.13\right)$ but very different EoR morphologies. The top panels correspond to our reference (ML) model, discussed already in Sec. 2.3. This model has $\alpha_{\mathrm{esc}}=-0.298$ and $M_{\text {turn }}=7.2 \times 10^{8} \mathrm{M}_{\odot}$, resulting in relatively massive galaxies driving the EoR; thus we label it Ref/BrightGalaxies. Below it we show a FaintGalaxies model with the following

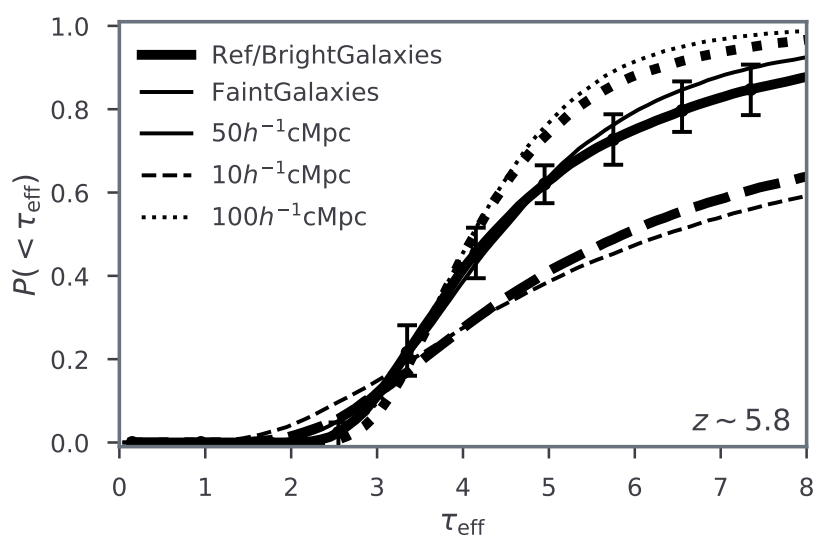

Figure 7. CDFs of $\tau_{\text {eff }}$ and $\ln \tau_{\text {eff }}$ at $z=5.8$ averaged over 50 , 10 and $100 h^{-1} \mathrm{cMpc}$ from Ref/BrightGalaxies (thick curves) and FaintGalaxies (thin curves). Uncertainties ([14, 86] percentiles) for the $50 \mathrm{~h}^{-1} \mathrm{cMpc}$ result in Ref/BrightGalaxies are indicated in a subset of the bins.

astrophysical parameters $\left(f_{*, 10}, \alpha_{*}, f_{\mathrm{esc}, 10}, \alpha_{\mathrm{esc}}, M_{\mathrm{turn}}, t_{*}\right)$ $=\left(0.084,0.483,0.019,-0.834,2.16 \times 10^{8} \mathrm{M}_{\odot}, 0.678\right)$. Note that we also include $f_{\text {rescale }}=0.12$ in the FaintGalaxies model to rescale the Ly $\alpha$ transmission at $z=5.8$ (see equation 9. compared to $f_{\text {rescale }}=0.92$ in BrightGalaxies). Although both models have the same HII filling factor at $z=5.8$, the EoR morphologies are noticeably different, as expected from the different biases of their corresponding dominant galaxy population. Specifically, FaintGalaxies is characterized with more numerous, smaller HII regions driven by its more abundant, yet fainter galaxies. As a result, the UVB is weaker, as the contribution of very distant sources is limited by the small sizes of the HII regions.

To quantify if the different EoR morphologies result in different $\tau_{\text {eff }}$ distributions, in Fig. 7 we compute the CDFs 
averaging over 10, 50 (the default value used in the MCMC), and $100 h^{-1} \mathrm{Mp}{ }^{19}$

From the figure we see that the difference in the CDFs, when normalized to have the same Lyman alpha transmission, is modest - smaller than the cosmic variance of the current sample (see the errorbar for the $50 h^{-1} \mathrm{cMpc}$ result in Ref/BrightGalaxies). This is due to the fact that the dynamic range probed by the forest is too small to discriminate against different EoR morphologies at a fixed neutral fraction. Indeed, we confirm that the largest differences between the two models, when averaged over $10 h^{-1} \mathrm{cMpc}$, occurs at $\tau_{\text {eff }} \sim 90$. This is far beyond the observable range. In matching the same mean flux, the differences in $\tau_{\text {eff }}$ appear in the tail of the PDF, and are thus difficult to constrain. A larger quasar sample $\left(N_{\text {sample }} \sim 1000\right)$ would narrow down the cosmic variance uncertainties within the accessible range; however, such a large sample might only become feasible with next-generation telescopes such as Euclid.

Therefore, we conclude that the current Ly $\alpha$ forest data is unlikely to be able to distinguish between different galaxy models having the same global properties (such as the EoR history; see also Nasir \& D'Aloisio 2020 for a similar conclusion). In future work, we will also forward model the Lyman$\beta$ forest, quantifying if its added dynamic range can further help constrain models (as has been implied by, e.g. Eilers et al. 2019, Keating et al. 2020a), despite the added cosmic variance from the overlapping, lower redshift Ly $\alpha$ forest. We will also investigate the constraining power of other summary statistics.

\subsection{Comparison to previous works}

Previous studies have made significant progress in understanding the large-scale Ly $\alpha$ opacity fluctuations at $z \gtrsim 5.5$. However, since forest simulations are generally computationally expensive, few studies do exhaustive parameter exploration. Instead, most focus on one or two of the following dominant sources of fluctuations - the UVB (either through rare sources or a short mean free path), the gas temperature, and late reionization. In this section we compare our maximum likelihood model from the forest MCMC (labeled ref) to some of the recent works that managed to explain the large-scale opacity fluctuations. In Fig. 8 we show the average EoR history, photoionization rate, ionizing emissivity/recombination rate and mean free path, from re ${ }^{20}$ along with some other models and observational data.

As demonstrated quantitatively in Section 2.3.3 the patchy UVB is an important source of forest fluctuations. However, we find that UVB fluctuations alone are not sufficient, with late reionization required to fully explain the data (see also, e.g. Keating et al. 2020a Choudhury et al.

19 We note that the FaintGalaxies model has a different EoR evolution and is disfavored by the total likelihood in forest. However, here we focus only on the CDFs at $z=5.8$ where the two models have the same neutral fraction and comparable likelihoods (i.e. $\mathscr{L}_{z=5.8}$; see Sec. 5.1 .

20 In principle, our Bayesian framework allows us to compare the posterior distributions of these quantities. However, we do not output all of these fields when running the MCMC. Therefore, here we only show the maximum likelihood model that was rerun to output the desired quantities.

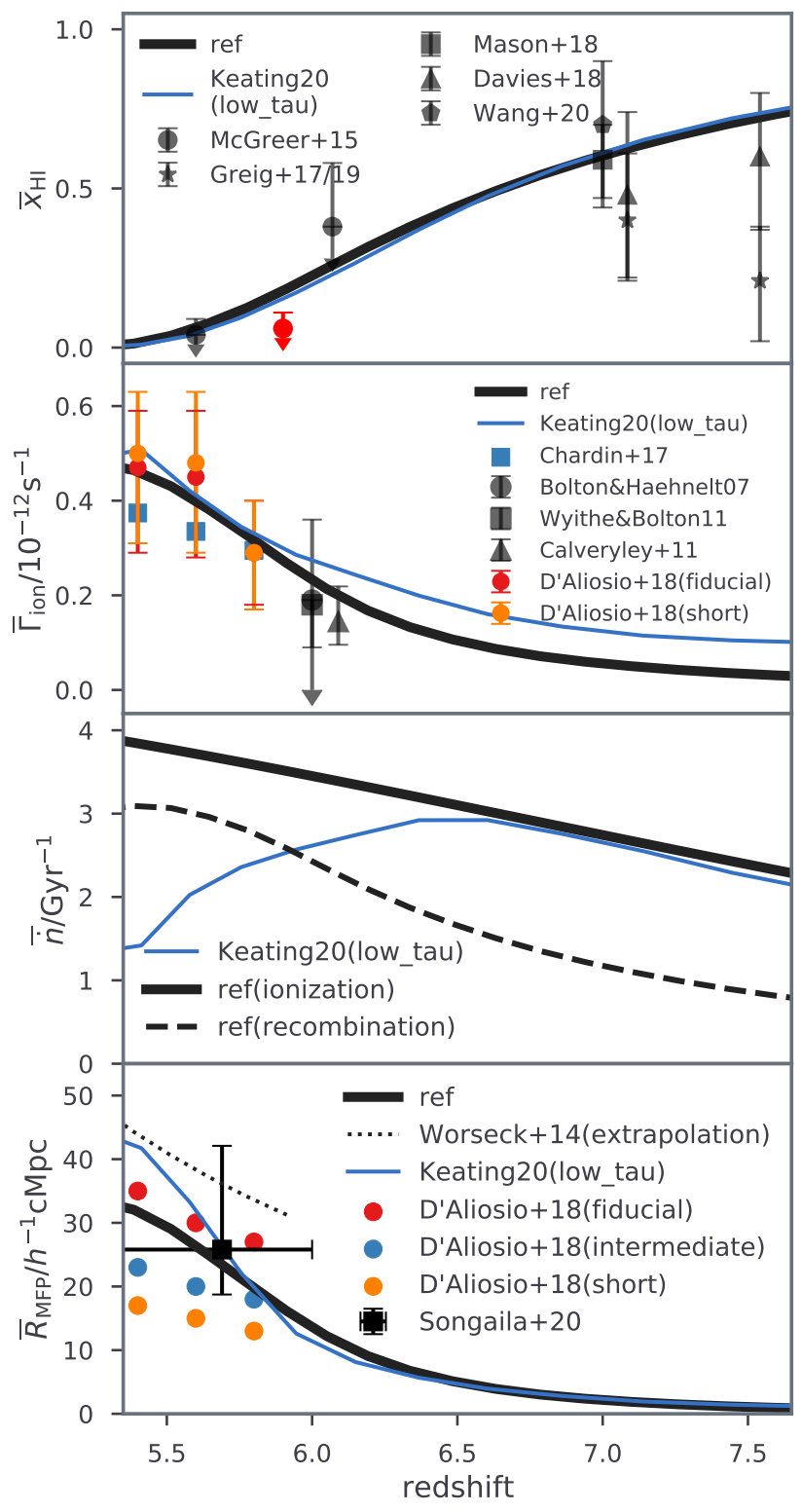

Figure 8. The average neutral hydrogen fraction, photoionization rate, ionizing emissivity/recombination rate, mean free path and gas temperature of our maximum likelihood parameter combination $(r e f)$, along with some other models (Chardin et al.|2017 D'Aloisio et al. 2018, Keating et al. 2020a) and observational limits (Bolton \& Haehnelt 2007b | Wyithe \& Bolton 2011| Calverley et al. 2011 | Worseck et al. 2014 | McGreer et al. 2015 Greig et al. 2017, 2019 Davies et al. 2018b Mason et al. 2018; Wang et al. 2020

2021). Some previous works have focused on the patchy UVB, sourced by either rare, bright AGN or a small mean free path. We briefly discuss each in turn.

Although rare, bright sources could explain the $\tau_{\text {eff }}$ CDF (Chardin et al. 2015, 2017, D'Aloisio et al. 2017 Meiksin 2020), they somewhat struggle to reproduce the longest GP trough found in ULAS J0148+0600, and would likely be in tension with IGM temperature measurements (e.g. D'Aloisio et al. 2017). Furthermore, extrapolations of AGN LFs and semi-analytic models suggest they provide a 
negligible contribution to the UVB at $z>5.5$ (e.g. Mitra et al. 2015, Manti et al. 2017, Parsa et al. 2017. Qin et al. 2017; Garaldi et al. 2019).

On the other hand, a small mean free path could modulate a galaxy-dominated UVB to the level required to explain the forest observations (e.g. Davies \& Furlanetto 2016 D'Aloisio et al. 2018). However, these models generally require very short mean free paths (c.f. the early-reionizationshort-mfp model from Nasir \& D'Aloisio 2020 and the short mean free path from D'Aloisio et al. 2018 denoted with the orange points in the bottom panel of Fig. 8). As discussed in D'Aloisio et al. (2018), observational estimates of the mean free path (e.g. Songaila \& Cowie 2010, Worseck et al. 2014) may be biased high, due to contamination from the proximity zone in which the QSO flux dominates over that of the UVB. Indeed, the mean free path from our ML model is in good agreement with the fiducial values computed in that work, but significantly above those required to explain the opacity fluctuations without evoking a late reionization. Unlike these works, our framework does not have a tuning knob for the mean free path, instead calculating this quantity directly from the source and sink distributions following Sobacchi \& Mesinger (2014). Our galaxy models and associated parameter priors do not result in mean free paths of $<10 \mathrm{cMpc}$ at $z<6$.

In addition to a patchy UVB, large-scale temperature fluctuations have been evoked to explain the forest observations (D'Aloisio et al. 2015). However, as discussed in Section 2.3.3 we find temperature fluctuations have only a minor impact (see also Keating et al. 2018). This could be due to the fact that strong temperature fluctuations require fairly extended reionization histories, which are disfavored in our posterior. Indeed, the fiducial model in D'Aloisio et al. (2015) is in mild tension with the latest Planck measurement (Planck Collaboration et al. 2020). Furthermore, these models require that troughs in the transmission come from large scale overdensities, which host an overabundance of galaxies and thus reionize early. This is contrary to recent observations showing a dearth of galaxies around the long GP trough of ULAS J0148+0600 (Becker et al. 2018, Kashino et al. 2020).

Finally, late reionization (e.g. Mesinger 2010) has also been used to explain the $\tau_{\text {eff }}$ distributions (Kulkarni et al. 2019, Keating et al. 2020a b, Nasir \& D'Aloisio 2020). Our results are consistent with this claim (see also the recent work by Choudhury et al. 2021). In Fig. 8 we show the fiducial model from Keating et al. (2020a), together with our maximum likelihood model. Amazingly, the EoR histories are in perfect agreement, despite the fact that Keating et al. (2020a) did not perform Bayesian inference. However, their model requires a drop in the ionizing emissivity $\left(\overline{\dot{n}}_{\text {ion }}\right)$ at $z<6.5$, which is difficult to justify physically and is in contradiction with the observed redshift evolution of the star formation rate density (e.g. Bouwens et al. 2015). On the other hand, our model achieves the same EoR history without a non-monotonic evolution of the emissivity; the recombination rate increases, approaching the ionization rate, resulting in relatively slow, "photon starved" end to reionization (e.g. Bolton \& Haehnelt 2007b. Sobacchi \& Mesinger 2014). One explanation for this difference might be that the simulations of Keating et al. (2020a), with a gas particle mass of $\sim 10^{7} M_{\odot}$, could be under-resolving small gas clumps and thus under-estimating the impact of recombinations. Although the mean free path (being an instantaneous, volume averaged quantity) does not directly show the cumulative impact of inhomogeneous recombinations, the rapid rise in their mean free path below $z<6$ seen in the bottom panel supports this explanation.

\section{CONCLUSIONS}

In this work, we extend the Bayesian inference framework of $21 \mathrm{cmFAST} / 21 \mathrm{CMMC}$ to forward-model the (low resolution) $\operatorname{Ly} \alpha$ forest. We run MCMCs by sampling empirical galaxy scaling relations and computing the corresponding $3 \mathrm{D}$ lightcones of the Ly $\alpha$ forest. With these, we quantify the additional constraining power provided by observations of large-scale opacity fluctuations (i.e. PDFs of $\tau_{\text {eff }}$ averaged over $50 \mathrm{cMpc} / h$; Bosman et al. 2018, 2020).

We find that, in order to be consistent with the observations, our models require late reionization. The final overlap stages of the EoR, corresponding to when $>95 \%$ of the volume was ionized, occur at $z<5.6$. Our Bayesian framework provides statistical proof of previous suggestions that the EoR might have completed reionization at $z<6$ (Lidz et al. 2007. Mesinger 2010, Kulkarni et al. 2019, Keating et al. 2020b; Nasir \& D'Aloisio 2020), and is perfectly consistent with the recent, similar analysis by Choudhury et al. (2021). Such late reionization is in mild tension $(\sim 1.5 \sigma)$ with the dark fraction upper limits from (McGreer et al. 2015). In the future, we will revisit this mild tension using updated data from the XQR-30 large VLT program.

We also find that the forest data improves our current knowledge of galaxy UV ionizing properties. In particular, we find a weak $(\sim 1 \sigma)$ constraint on the turn-over halo mass scale $\left(M_{\text {turn }}=2 \times 10^{8}-10^{9} \mathrm{M}_{\odot}\right)$, below which star formation stops being efficient. Moreover, we find that the late reionization preferred by the forest data tightens constraints on the ionizing escape fraction. Combined observations (i.e. galaxy UV LFs, CMB optical depth, dark fraction and forest) favor a characteristic ionizing escape fraction of $f_{\text {esc }}=6.9_{-2.6}^{+3.8} \%$, and disfavor a strong evolution with the galaxy's halo (or stellar) mass. Unfortunately, the $\tau_{\text {eff }}$ CDFs cannot distinguish among different source/sink models that have different EoR morphologies but the same EoR history.

Using our maximum-likelihood model, we demonstrate that large-scale opacity fluctuations are driven by a combination of both patchy reionization and spatial variations in the photoionizing background (with temperature inhomogenities being sub-dominant). The cosmic Hi patches and regions of weak UVB both corresponds to large-scale underdensities in the matter field. Thus the longest GunnPeterson (GP) troughs correlate with a relative dearth of galaxies, in agreement with observations (Becker et al. 2018, Kashino et al. 2020) and some previous models (e.g. Davies et al. 2018a).

Our inference framework can easily be extended to include different source models, such as AGN and/or having a more complex parametrization of galaxy evolution. Using the Bayesian evidence, we can quantify if the data require the additional model complexity (e.g. Qin et al. 2020a). We postpone such investigation to future work, applying them on upcoming, larger data sets. 


\section{ACKNOWLEDGEMENTS}

We thank George Becker, Anson D'Aloisio, Frederick Davies, Laura Keating, and Girish Kulkarni for helpful comments on a draft version of this paper. This work was supported by the European Research Council (ERC) under the European Union's Horizon 2020 research and innovation programmes (AIDA - \#638809, First Light - \#669253 and Cosmic Gas - \#740246). The results presented here reflect the authors' views; the ERC is not responsible for their use. We acknowledge computational resources of the HPC center at SNS. MV is supported by grants INFN INDARK PD51 and ASI-INAF n.2017-14-H.0.

\section{DATA AVAILABILITY}

The data underlying this article will be shared on reasonable request to the corresponding author.

\section{REFERENCES}

Ahn K., Shapiro P. R., 2020, arXiv e-prints, arXiv:2011.03582

Atek H., Richard J., Kneib J.-P., Schaerer D., 2018, MNRAS, 479, 5184

Bañados E. et al., 2018, Nature, 553, 473

Barkana R., Loeb A., 2005, ApJ, 626, 1

Bauer A., Springel V., Vogelsberger M., Genel S., Torrey P., Sijacki D., Nelson D., Hernquist L., 2015, MNRAS, 453, 3593

Bautista J. E. et al., 2017, A\&A, 603, A12

Becker G. D., Bolton J. S., 2013, MNRAS, 436, 1023

Becker G. D., Bolton J. S., Madau P., Pettini M., RyanWeber E. V., Venemans B. P., 2015, MNRAS, 447, 3402

Becker G. D., Davies F. B., Furlanetto S. R., Malkan M. A., Boera E., Douglass C., 2018, ApJ, 863, 92

Becker G. D., Rauch M., Sargent W. L. W., 2007, ApJ, 662,72

Behroozi P., Wechsler R. H., Hearin A. P., Conroy C., 2019, MNRAS, 488, 3143

Bhatawdekar R., Conselice C. J., Margalef-Bentabol B., Duncan K., 2019, MNRAS, 486, 3805

Bolton J. S., Becker G. D., Wyithe J. S. B., Haehnelt M. G., Sargent W. L. W., 2010, MNRAS, 406, 612

Bolton J. S., Haehnelt M. G., 2007a, MNRAS, 374, 493

Bolton J. S., Haehnelt M. G., 2007b, MNRAS, 382, 325

Bolton J. S., Haehnelt M. G., Warren S. J., Hewett P. C., Mortlock D. J., Venemans B. P., McMahon R. G., Simpson C., 2011, MNRAS, 416, L70

Bolton J. S., Puchwein E., Sijacki D., Haehnelt M. G., Kim T.-S., Meiksin A., Regan J. A., Viel M., 2017, MNRAS, 464,897

Bosman S., 2020, in Zenodo dataset, Vol. 36, p. 34964

Bosman S. E. I., Fan X., Jiang L., Reed S., Matsuoka Y., Becker G., Haehnelt M., 2018, MNRAS, 479, 1055

Bosman S. E. I., Ďurovčíková D., Davies F. B., Eilers A. C., 2020, arXiv e-prints, arXiv:2006.10744

Bouwens R. J., Illingworth G. D., Oesch P. A., Caruana J., Holwerda B., Smit R., Wilkins S., 2015b, ApJ, 811, 140

Bouwens R. J. et al., 2015, ApJ, 803, 34
Bouwens R. J., Oesch P. A., Illingworth G. D., Ellis R. S., Stefanon M., 2017, ApJ, 843, 129

Bouwens R. J. et al., 2016, ApJ, 830, 67

Busca N. G. et al., 2013, A\&A, 552, A96

Calverley A. P., Becker G. D., Haehnelt M. G., Bolton J. S., 2011, MNRAS, 412, 2543

Chardin J., Haehnelt M. G., Aubert D., Puchwein E., 2015, MNRAS, 453, 2943

Chardin J., Puchwein E., Haehnelt M. G., 2017, MNRAS, 465,3429

Choudhury T. R., Haehnelt M. G., Regan J., 2009, MNRAS, 394, 960

Choudhury T. R., Paranjape A., Bosman S. E. I., 2021, MNRAS

Croft R. A. C., Weinberg D. H., Bolte M., Burles S., Hernquist L., Katz N., Kirkman D., Tytler D., 2002, ApJ, 581, 20

D'Aloisio A., McQuinn M., Davies F. B., Furlanetto S. R., 2018, MNRAS, 473, 560

D'Aloisio A., McQuinn M., Maupin O., Davies F. B., Trac H., Fuller S., Upton Sanderbeck P. R., 2019, ApJ, 874, 154

D'Aloisio A., McQuinn M., Trac H., 2015, ApJ, 813, L38

D'Aloisio A., Upton Sanderbeck P. R., McQuinn M., Trac H., Shapiro P. R., 2017, MNRAS, 468, 4691

Davies F. B., Becker G. D., Furlanetto S. R., 2018a, ApJ, 860, 155

Davies F. B., Furlanetto S. R., 2016, MNRAS, 460, 1328

Davies F. B. et al., 2018b, ApJ, 864, 142

Davies F. B., Hennawi J. F., Eilers A.-C., 2020, MNRAS, 493, 1330

Davies J. E., Mutch S. J., Qin Y., Mesinger A., Poole G. B., Wyithe J. S. B., 2019, MNRAS, 489, 977

Delubac T. et al., 2015, A\&A, 574, A59

Dixon K. L., Iliev I. T., Mellema G., Ahn K., Shapiro P. R., 2016, MNRAS, 456, 3011

Efstathiou G., 1992, MNRAS, 256, 43P

Eilers A.-C., Davies F. B., Hennawi J. F., 2018, ApJ, 864, 53

Eilers A.-C., Davies F. B., Hennawi J. F., Prochaska J. X., Lukić Z., Mazzucchelli C., 2017, ApJ, 840, 24

Eilers A.-C., Hennawi J. F., Davies F. B., Oñorbe J., 2019, ApJ, 881, 23

Eilers A.-C. et al., 2020, ApJ, 900, 37

Fan X. et al., 2006, AJ, 132, 117

Faucher-Giguère C.-A., Lidz A., Hernquist L., Zaldarriaga M., 2008a, ApJ, 682, L9

Faucher-Giguère C.-A., Prochaska J. X., Lidz A., Hernquist L., Zaldarriaga M., 2008b, ApJ, 681, 831

Ferrara A., Loeb A., 2013, MNRAS, 431, 2826

Finkelstein S. L. et al., 2015, ApJ, 810, 71

Friedrich M. M., Mellema G., Alvarez M. A., Shapiro P. R., Iliev I. T., 2011, MNRAS, 413, 1353

Furlanetto S. R., Zaldarriaga M., Hernquist L., 2004, ApJ, 613,1

Gaikwad P. et al., 2020, MNRAS, 494, 5091

Gallerani S., Ferrara A., Fan X., Choudhury T. R., 2008, MNRAS, 386, 359

Garaldi E., Compostella M., Porciani C., 2019, MNRAS, 483, 5301

Garzilli A., Bolton J. S., Kim T. S., Leach S., Viel M., 2012, MNRAS, 424, 1723 
Greig B., Mesinger A., 2015, MNRAS, 449, 4246

Greig B., Mesinger A., 2017, MNRAS, 472, 2651

Greig B., Mesinger A., 2018, MNRAS, 477, 3217

Greig B., Mesinger A., Bañados E., 2019, MNRAS, 484, 5094

Greig B., Mesinger A., Haiman Z., Simcoe R. A., 2017, MNRAS, 466, 4239

Gunn J. E., Peterson B. A., 1965, ApJ, 142, 1633

Haardt F., Madau P., 2012, ApJ, 746, 125

Hopkins P. F., Kereš D., Oñorbe J., Faucher-Giguère C.A., Quataert E., Murray N., Bullock J. S., 2014, MNRAS, 445,581

Hopkins P. F. et al., 2018, MNRAS, 480, 800-863

Hui L., Gnedin N. Y., 1997, MNRAS, 292, 27

Hutter A., 2018, MNRAS, 477, 1549

Hutter A., Dayal P., Müller V., Trott C. M., 2017, ApJ, 836,176

Iliev I. T., Mellema G., Pen U. L., Merz H., Shapiro P. R., Alvarez M. A., 2006, MNRAS, 369, 1625

Iršič V. et al., 2017, MNRAS, 466, 4332

Ishigaki M., Kawamata R., Ouchi M., Oguri M., Shimasaku K., Ono Y., 2018, ApJ, 854, 73

Jiang L. et al., 2016, ApJ, 833, 222

Kashino D., Lilly S. J., Shibuya T., Ouchi M., Kashikawa N., 2020, ApJ, 888, 6

Keating L. C., Kulkarni G., Haehnelt M. G., Chardin J., Aubert D., 2020a, MNRAS, 497, 906

Keating L. C., Puchwein E., Haehnelt M. G., 2018, MNRAS, 477, 5501

Keating L. C., Weinberger L. H., Kulkarni G., Haehnelt M. G., Chardin J., Aubert D., 2020b, MNRAS, 491, 1736

Kimm T., Cen R., 2014, ApJ, 788, 121

Kuhlen M., Faucher-Giguère C.-A., 2012, MNRAS, 423, 862

Kulkarni G., Keating L. C., Haehnelt M. G., Bosman S. E. I., Puchwein E., Chardin J., Aubert D., 2019, MNRAS, 485, L24

Lee K.-G. et al., 2013, AJ, 145, 69

Lee K.-G., Cen R., Gott, J. Richard I., Trac H., 2008, ApJ, 675,8

Lee K.-G. et al., 2015, ApJ, 799, 196

Lee K.-G. et al., 2014, ApJ, 795, L12

Lidz A., Faucher-Giguère C.-A., Dall'Aglio A., McQuinn M., Fechner C., Zaldarriaga M., Hernquist L., Dutta S., 2010, ApJ, 718, 199

Lidz A., McQuinn M., Zaldarriaga M., Hernquist L., Dutta S., 2007, ApJ, 670, 39

Livermore R. C., Finkelstein S. L., Lotz J. M., 2017, ApJ, 835,113

Ma X., Kasen D., Hopkins P. F., Faucher-Giguère C.-A., Quataert E., Kereš D., Murray N., 2015, MNRAS, 453, 960

Ma X., Quataert E., Wetzel A., Hopkins P. F., FaucherGiguère C.-A., Kereš D., 2020, MNRAS, 498, 2001

Madau P., Dickinson M., 2014, Annual Review of Astronomy and Astrophysics, 52, 415

Madau P., Haardt F., 2015, ApJ, 813, L8

Manti S., Gallerani S., Ferrara A., Greig B., Feruglio C., 2017, MNRAS, 466, 1160

Maselli A., Gallerani S., Ferrara A., Choudhury T. R., 2007, MNRAS, 376, L34

Mason C. A., Treu T., Dijkstra M., Mesinger A., Trenti M.,
Pentericci L., de Barros S., Vanzella E., 2018, ApJ, 856, 2 McGreer I. D., Mesinger A., D'Odorico V., 2015, MNRAS, 447, 499

McQuinn M., Lidz A., Zahn O., Dutta S., Hernquist L., Zaldarriaga M., 2007, MNRAS, 377, 1043

McQuinn M., Upton Sanderbeck P. R., 2016, MNRAS, 456, 47

Meiksin A., 2020, MNRAS, 491, 4884

Mesinger A., 2010, MNRAS, 407, 1328

Mesinger A., ed., 2016, Understanding the Epoch of Cosmic Reionization, Vol. 423. Springer International Publishing Switzerland

Mesinger A., ed., 2019, The Cosmic 21-cm Revolution. IOPPublishing, Bristol, UK, pp. 2514-3433

Mesinger A., Furlanetto S., 2007, ApJ, 669, 663

Mesinger A., Furlanetto S., 2009, MNRAS, 400, 1461

Mesinger A., Furlanetto S., Cen R., 2011, MNRAS, 411, 955

Mesinger A., Greig B., Sobacchi E., 2016, MNRAS, 459, 2342

Mesinger A., Haiman Z., Cen R., 2004, ApJ, 613, 23

Miralda-Escudé J., Cen R., Ostriker J. P., Rauch M., 1996, ApJ, 471, 582

Miralda-Escudé J., Haehnelt M., Rees M. J., 2000, ApJ, 530,1

Mitra S., Roy Choudhury T., Ferrara A., 2015, Monthly Notices of the Royal Astronomical Society: Letters, 454, L76

Mortlock D. J. et al., 2011, Nature, 474, 616

Moster B. P., Naab T., White S. D. M., 2013, MNRAS, 428, 3121

Murray S. G., Greig B., Mesinger A., Muñoz J. B., Qin Y., Park J., Watkinson C. A., 2020, Journal of Open Source Software, 5, 2582

Mutch S. J., Geil P. M., Poole G. B., Angel P. W., Duffy A. R., Mesinger A., Wyithe J. S. B., 2016, MNRAS, 462, 250

Nasir F., D'Aloisio A., 2020, MNRAS, 494, 3080

Oesch P. A., Bouwens R. J., Illingworth G. D., Labbé I., Stefanon M., 2018, ApJ, 855, 105

Oesch P. A. et al., 2016, ApJ, 819, 129

Paardekooper J.-P., Khochfar S., Dalla Vecchia C., 2015, MNRAS, 451, 2544

Paranjape A., Choudhury T. R., 2014, MNRAS, 442, 1470

Park J., Mesinger A., Greig B., Gillet N., 2019, MNRAS, 484, 933

Parsa S., Dunlop J. S., McLure R. J., 2017, MNRAS, 474, 2904-2923

Planck Collaboration et al., 2016, A\&A, 594, A13

Planck Collaboration et al., 2020, A\&A, 641, A6

Price L. C., Trac H., Cen R., 2016, arXiv e-prints, arXiv:1605.03970

Puchwein E., Bolton J. S., Haehnelt M. G., Madau P., Becker G. D., Haardt F., 2015, MNRAS, 450, 4081

Qin Y., Mesinger A., Greig B., Park J., 2020a, MNRAS, staa3408

Qin Y. et al., 2017, MNRAS, 472, 2009-2027

Qin Y., Poulin V., Mesinger A., Greig B., Murray S., Park J., 2020b, MNRAS, 499, 550

Rahmati A., Pawlik A. H., Raičević M., Schaye J., 2013, MNRAS, 430, 2427

Rauch M., 1998, ARA\&A, 36, 267 
Robertson B. E., Ellis R. S., Furlanetto S. R., Dunlop J. S., 2015, ApJ, 802, L19

Sadoun R., Zheng Z., Miralda-Escudé J., 2017, ApJ, 839, 44

Savaglio S., Panagia N., Padovani P., 2002, ApJ, 567, 702

Schaye J., 2001, ApJ, 559, 507

Schroeder J., Mesinger A., Haiman Z., 2013, MNRAS, 428, 3058

Scoccimarro R., 1998, MNRAS, 299, 1097

Shapiro P. R., Giroux M. L., Babul A., 1994, ApJ, 427, 25

Slosar A. et al., 2013, J. Cosmology Astropart. Phys., 2013, 026

Sobacchi E., Mesinger A., 2014, MNRAS, 440, 1662

Songaila A., Cowie L. L., 2010, ApJ, 721, 1448

Springel V. et al., 2005, Nature, 435, 629

Sun G., Furlanetto S. R., 2016, MNRAS, 460, 417

Tacchella S., Bose S., Conroy C., Eisenstein D. J., Johnson

B. D., 2018, ApJ, 868, 92

Thoul A. A., Weinberg D. H., 1996, ApJ, 465, 608

Trac H. Y., Gnedin N. Y., 2011, Advanced Science Letters, 4, 228

Venemans B. P. et al., 2013, ApJ, 779, 24

Viel M., Becker G. D., Bolton J. S., Haehnelt M. G., 2013, Phys. Rev. D, 88, 043502

Viel M., Haehnelt M. G., 2006, MNRAS, 365, 231

Viel M., Lesgourgues J., Haehnelt M. G., Matarrese S., Riotto A., 2005, Phys. Rev. D, 71, 063534

Wang F. et al., 2020, ApJ, 896, 23

Weinberg D., et al., 1999, in Evolution of Large Scale Structure : From Recombination to Garching, Banday A. J., Sheth R. K., da Costa L. N., eds., p. 346

Willott C. J. et al., 2010, AJ, 139, 906

Worseck G. et al., 2014, MNRAS, 445, 1745

Wu X.-B. et al., 2015, Nature, 518, 512

Wyithe J. S. B., Bolton J. S., 2011, MNRAS, 412, 1926

Wyithe J. S. B., Loeb A., 2013, MNRAS, 428, 2741

Xu H., Wise J. H., Norman M. L., Ahn K., O'Shea B. W., 2016, ApJ, 833, 84

Yang J. et al., 2020, ApJ, 897, L14

Yèche C., Palanque-Delabrouille N., Baur J., du Mas des Bourboux H., 2017, J. Cosmology Astropart. Phys., 2017, 047

Yung L. Y. A., Somerville R. S., Popping G., Finkelstein S. L., Ferguson H. C., Davé R., 2019, MNRAS, 490, 2855 Zahn O., Lidz A., McQuinn M., Dutta S., Hernquist L., Zaldarriaga M., Furlanetto S. R., 2007, ApJ, 654, 12

Zahn O., Mesinger A., McQuinn M., Trac H., Cen R., Hernquist L. E., 2011, MNRAS, 414, 727

Zeng C., Hirata C. M., 2020, arXiv e-prints, arXiv:2007.02940

\section{APPENDIX A: TESTING THE FLUCTUATING GUNN-PETERSON APPROXIMATION}

By computational necessity, our forward models of the Lyman alpha forest are low resolution $(\sim 2 \mathrm{cMpc}$ cells $)$, and use the fluctuating Gunn-Peterson approximation (FGPA). The lack of small-scale structure in our models could also impact the large-scale opacity fluctuations we use as our summary statistic (see, e.g. Viel \& Haehnelt 2006 for an example of the impact on the flux power spectrum at lower redshifts where the transmission is higher). In order to account for this source of inaccuracy, here we compute an error covariance matrix, using a high-resolution hydro simulation from the Sherwood suite (Bolton et al. 2017).

The simulation used in this section was run with an updated version of GADGET-2 (Springel et al. 2005 and assumes a $\Lambda$ CDM cosmology with parameters $\left(\Omega_{\mathrm{m}}, \Omega_{\mathrm{b}}, \Omega_{\Lambda}, h, \sigma_{8}, n_{s}=0.31,0.048,0.69,0.68,0.83,0.96\right)$ from Planck Collaboration et al. (2016). It includes $512^{3}$ baryonic and $512^{3}$ dark matter particles within a cube of $80 h^{-1} \mathrm{cMpc}$ on a side. The forest is calculated on a $2048^{3}$ Eulerian grid, with a corresponding resolution of $0.057 \mathrm{cMpc}$ (i.e. $80 h^{-1} \mathrm{cMpc} / 2048$ ).

We compute Ly $\alpha$ spectra and $\tau_{\text {eff }}$ PDFs (i) assuming the FGPA on fields smoothed down to $\sim 2 \mathrm{cMpc}$ resolution; and (ii) using the native high-resolution fields $(0.057 \mathrm{cMpc})$, including peculiar velocities, and a Voigt profile for the Ly $\alpha$ absorption. The former corresponds to approximations used in our forward-models, while the latter we take as the "true" spectra.

We present the results in Fig. A1 including the hydrogen density, peculiar velocity, gas temperature, residual neutral hydrogen fraction, and the inferred Ly $\alpha$ optical depth as well as its transmission after rescaling the mean flux to the observed one (i.e. 0.0765; Bosman et al. 2020). Since the hydro simulation does not include patchy reionization, we focus on the $z=5.4$ snapshot (this is at the lowest end of the redshift range of interest, where patchy reionization should have the smallest impact; see Fig. 4 ).

As expected, having a lower resolution reduces the small-scale structure in the forest. Compared to the highresolution forest, the FGPA has a smaller variance on smallscales and $\mathrm{a} \sim 30 \%$ larger $f_{\text {rescale }}$ is required to match the same mean flux.

However, the differences are much smaller in the $\tau_{\text {eff }}$ CDFs, averaged over $50 h^{-1} \mathrm{cMpc}$. These are shown in the top panel of Fig. A2 for both the high-resolution spectra and the FGPA. We include also the observational data in gray. As expected, the simulation cannot match the observed distributions, owing to its small size and uniform UVB. However, the fact that the FGPA and the high-resolution spectra can produce comparable large-scale opacity fluctuations is highly encouraging of our approach.

In Appendix $B$ we show the corresponding error covariance matrix. This error is added to the cosmic variance error used when computing the forest likelihood in our MCMCs.

\section{APPENDIX B: ERROR COVARIANCE MATRICES}

We present the error covariance matrix ${ }^{21}$ (ECM) of the $\tau_{\text {eff }}$ PDF introduced by making the fluctuating Gunn-Peterson approximation (FGPA) or from the cosmic variance (CV) at $z=5.4-6$ in Fig. B1. Since ECMs are symmetric, we only show half of the FGPA and CV matrices, and present them together (i.e. top is FGPA and bottom is CV, respectively).

21 An identity matrix with a normalization of $10^{-5}$ is imposed on the total error covariance matrix as a precautionary measure. 

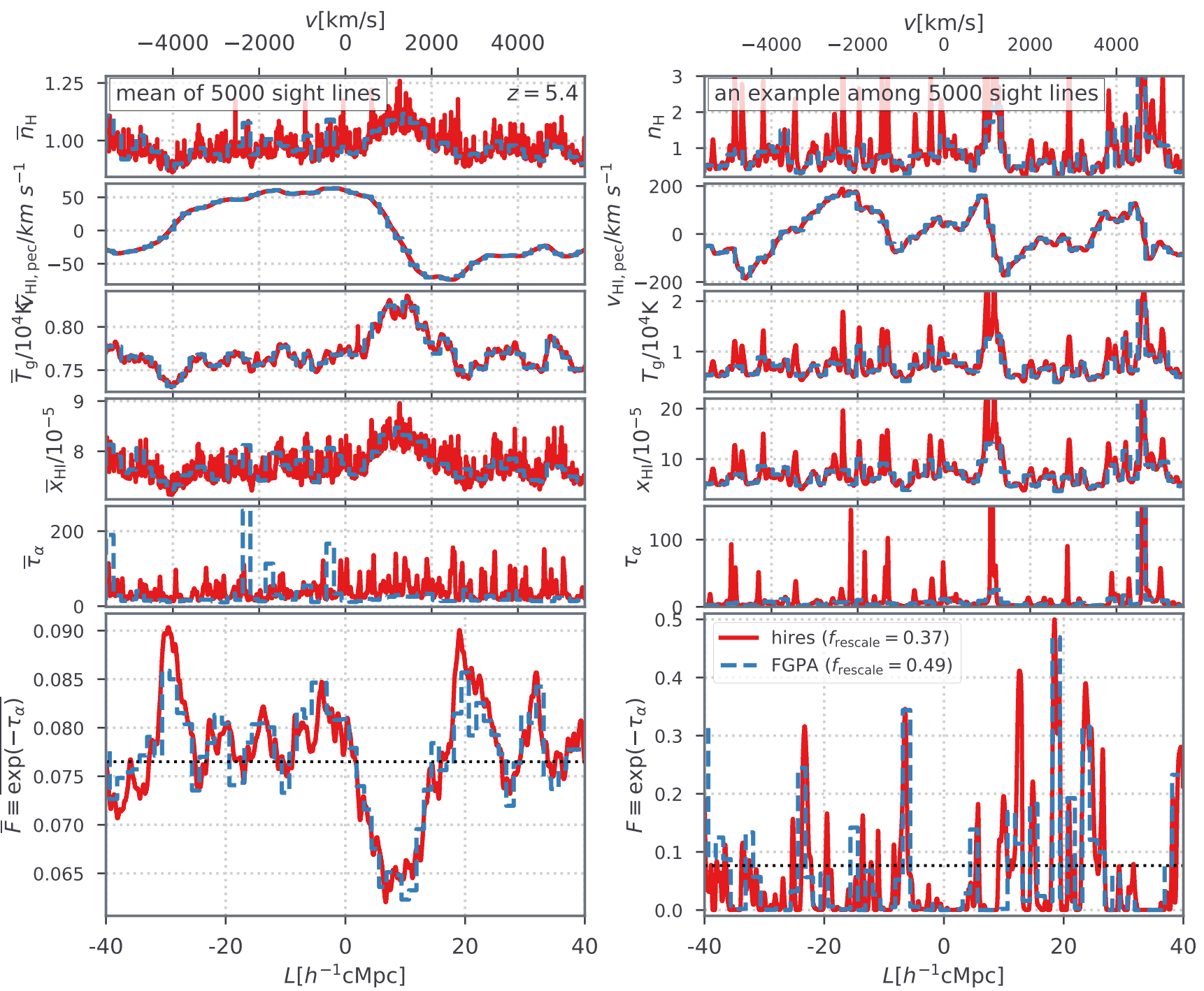

Figure A1. Properties including, from top to bottom, gas density, peculiar velocity, gas temperature, residual neutral hydrogen fraction, Ly $\alpha$ optical depth and its transmission after rescaling the mean flux to the observed one (indicated by the horizontal dotted lines; Bosman et al. 2020), along 5000 lines of sight across the entire Sherwood simulation box $\left(80 h^{-1} \mathrm{cMpc}\right)$ at $z=5.4$. Note that the mean of all sightlines are presented on the left while the right ones show one example sightline. The red solid curves indicate results from the reference model having high spectral resolution and integrating over the full Ly $\alpha$ profile when evaluating its optical depth (hires). The blue dashed lines correspond to results assuming FGPA and a lower resolution similar to what is used in the main context (FGPA).

For this plot, the $\mathrm{CV}$ is estimated using the 500 realizations ${ }^{22}$ drawn from our ML model (see more in Section 2). We note that the cosmic variance ECM is re-computed on the fly for each sample in our MCMC; unfortunately, it is computationally impractical to do this also for the FGPA ECM 23 The latter was computed as described in the previ-

22 For computing efficiency, only 150 realizations are generated during the MCMC.

23 As we do not know a-priory what are the true values of these fields in the real Universe, the FGPA error covariance matrix should be recomputed for each forward modelled universe. In other words, we use $\Sigma_{\mathrm{GP}}\left(\theta=\theta_{\text {Sherwood }}\right)$ in our MCMC, where $\theta_{\text {Sherwood }}$ encapsulates all of the choices and approximations made to generate the Sherwood simulation. However, we should instead know the general error covariance, $\Sigma_{\mathrm{GP}}(\theta)$, evaluating it on-the-fly for any astrophysical parameter combination $\theta$. Un- ous Appendix at $z=5.4$, and adjusted for higher redshifts by shifting the PDF such that the mean flux matches the observations (see the corresponding CDFs in Fig. A2,

There are both positive (correlation; red) and negative values (anti-correlation; blue) between pairs of different $\tau_{\text {eff }}$ bins, with more showing anti-correlation when being closer to the diagonal (i.e. nearby bins). This is expected as increasing one histogram bin can be roughly compensated by a decrease in a nearby bin. The dominant component in the total ECM is caused by the diagonal coefficients of the FGPA, though the and its coefficients along the diagonal (i.e.

fortunately, this is computationally impractical. Using different hydro simulations, in the future we will explore how the covariance matrix changes for a few different values of $\theta$, and estimate the corresponding impact on the posteriors. 


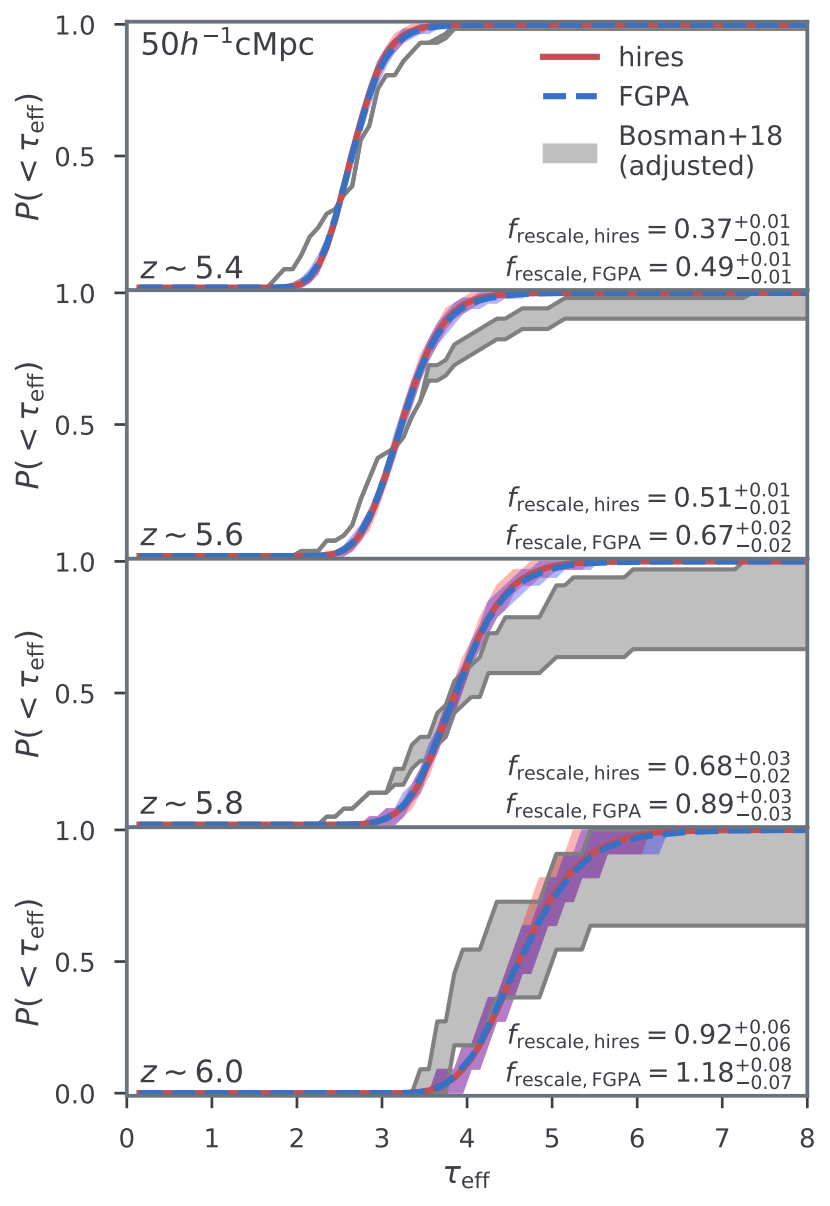

Figure A2. The CDF of $\tau_{\text {eff }}$ (averaged over $50 h^{-1} \mathrm{cMpc}$ ) at $z=5.4-6$ from the high-resolution Sherwood hydrodynamical simulation (hires; red) and assuming FGPA with low resolution ( $F G P A$; blue). The colored lines with shaded region indicate the mean and $[14,86]$ percentiles drawn from 500 realizations. The observed CDFs are indicated in grey (see more in Section 3).

uncertainties in each $\tau_{\text {eff }}$ bin of the PDF) decreases towards lower and higher redshifts. This is because when the sample size decreases, the PDF, with a lower value, also possesses a smaller absolute difference between the FGPA and the reference results (e.g. see the corresponding CDF in the lower right panel of Fig. A1). On the other hand, the CV ECM shows the opposite trend, which is also caused by the sample size. Since different lines of sight are randomly selected when estimating the cosmic variance, a smaller sample size leads to larger differences between different realizations and therefore larger $\mathrm{CV}$.

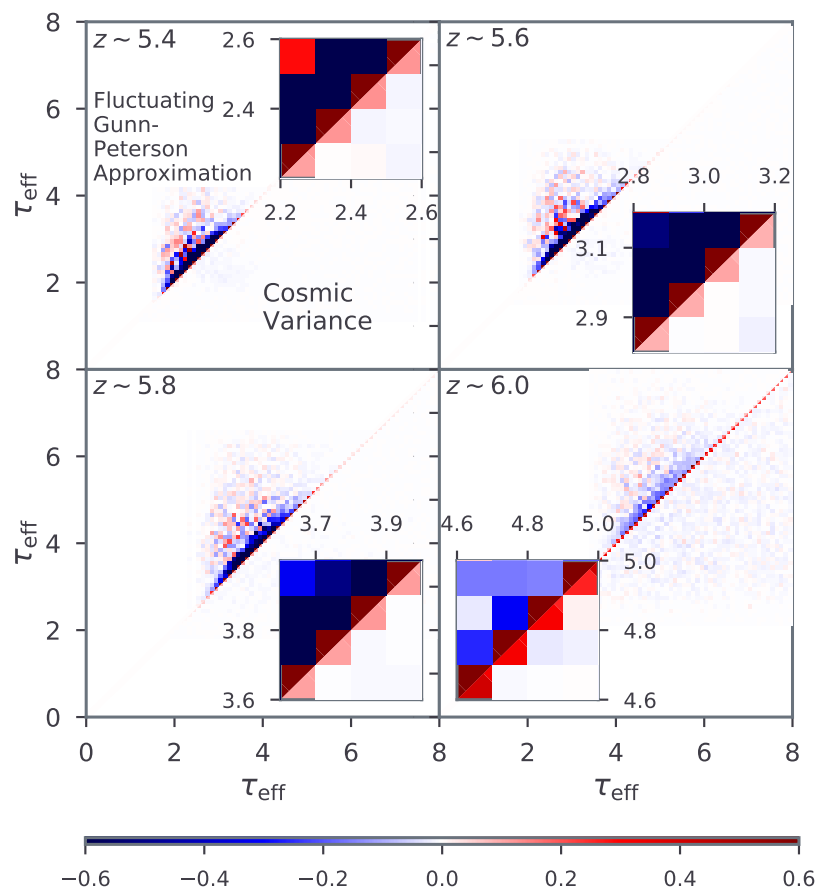

Figure B1. The error covariance matrices (ECMs) of the $\tau_{\text {eff }}$ PDF introduced by the fluctuating Gunn-Peterson approximation (FGPA) and cosmic variance (CV) at $z=5-6$. The two symmetric matrices are presented together in each sub-panel separated by the diagonal. The coefficients of the ECMs are represented by varying colors shown in the colorbar. A zoom-in sub-panel is presented to show more details around the maximum diagonal element of either matrix. 